\title{
Joachim Nocke Autopoiesis - Rechtssoziologie in seltsamen Schleifen
}

\author{
1. Woher kommt das systemtheoreusche Wissen?
}

Wer sich heure aus seinem juristischen Allragsgeschäft aufmacht, um in den Grundlagen seiner Disziplin cinmal wieder nach dem Rechten zu schauen, mag alsbald wieder verzag̨ nach Hause drängen. Denn schon beim Eintrict wird ihm der Boden unter den Füßen weggezogen. Die Begriffe von einst tragen nicht mehr. Nie gehörre Laute sind an ihre Stelle getreten. Autopoiesis, Bifurkation, Sacculinisierung und andere Wormirakel verschlagen ihm die Sprache. Auch das hier tätige Personal har sich merkivürdig verändert. Alte Bekannte, die vordem im Frankfurter Dialekr heimisch waren, sprechen heute den Systemjargon so fließend wie andere Leute Spanisch. Wieder andere, die sich gestern noch mit der Rechesnatur des behördlichen Essensgeldzuschusses quälten, haben Anschluß an die internacionale Gehirnforschung gefunden, zirieren unerschrocken mathematische Kongreßberichte, machen Vorschläge, wie man Kenntnisse der subatomaren Physik für die juristische Dogmatik „fruchrbar machen könnte . Unvernücks sieht er sich von Enzyklopädisten umstelht, wic man sie seir Leibniz, den sie souverän in die Schranken weisen, ausgestorben wähnte. Unhospitabel ist es hier geworden. Und keiner wird es dem Gast verübeln, wenn er eilig das Weite sucht. Hat er sich schnell um ein intellektuelles Abentever gebracht oder ist er vor kraftlosen Gespenstern geflohen, unter deren Bertlaken nur harmlose Bazonoi lärmten?

Es gehört zu den wissenschaftlichen Tugenden, sich gegenüber allen Aussagen der Wissenschaft prinzipiell kritisch zu verhalten. Je herrschender eine Meinung, je ambitionierter eine Theorie, desto wichtiger wird dieses Mißtrauen. In der Rechtsdogmarik verhält es sich bekanntlich umgekehrt. Je herrschender eine Meinung, desto weniger Widerspruch wird geduldet und folglich geübt. Die entscheidende Frage, die in unserem Zusammenhang zu stellen ist, ist daher eine Selbstverständlichkeit. Um so erstaunlicher, daß sie in den Kreisen, in denen wir uns nunmehr bewegen, selten gestellt wird: Woher bezieht die systemtheorerische Rechtssoziologie - um die es in diesem Beitrag ausschlielßlich geht - eigentlich ihr Wissen? Aus welchem Material, mit welchen Methoden und mit welcher Erkenntnistheorie schafft sie es, in rastloser Eile, ohne zu stocken, gewissermaßen fließbandartig Aussagen über die Gesellschaft und ihr Reche zu produzjeren? Gerade wenn man der Konzeprion, auf die sie sich beruft, gerecht werden will und nicht von vornherein dem Vorurteil nachgeben möchte, es handele sich bei dieser Theorie um eine Art Durchlauferhitzer, muß man mit Martin Lurher darauf bestehen: Woher kömmt ihnen diese Wissenschaft?

: Ich habe Leonie Brcunung für ihre Mithilie bel der Übersezzung der Systemtheone zu danken. 
Ein erster Eindruck will scheinen lassen: aus den Fußnoten. Das klingr nach einem erwas matten Scherz, denn wie sollen Wissenschaftler sonst belegen, woher sie ihr Wissen beziehen, wenn sie nicht in der glücklichen Lage sind, eigene empirische Forschung oder theoretische Überlegungen im Texc vorzuführen? Aber der Scherz ist keiner. Systemtheoretiker verweisen, wenn sie überhaupt auf jene Frage kommen, gewöhnlich auf andere Systemrheoretiker, bis dieser Verweisungskreislauf hierzulande - im Hinweis auf Luhmann vorläufig zum Stillstand zu kommen scheint. Aber auf wen verweist diese Autorität? Im Ziveifel - auf sich selbst. Diese Eigentümlichkeit erinnert an die Mechanismen juristischer Wissensprodukcion: man zitiert sich wechselseitig so lange, bis sich Meinungen zu Erkenntnissen verdichtet haben oder aber eine zentrale Figur das Machrwort spricht. Die "systemtheoretische" (zu den notwendigen Differenzierungen später) Zitiertechnik hat zweifellos auch erwas hiervon. Unabhängig hiervon drückt sich aber auch erwas anderes aus. Man hält sich des Zwangs zur Rechenschaft über den wissenschaftlichen Sratus und dje Methode seiner Erkenntnisgewinnung für enchoben. Entsprechende Fragen werden als faktisch entschieden behandelt. Es genügt ein Hinweis auf die Klassiker, um dann umstandslos zur Sache zu gehen.

An dieser Technik wäre nichts auszusetzen, wenn in der Wissenschaft die Fronten carsäclslich in dieser Weise geklär wären. Man kann nicht in jedem Aufsacz am Punkte Null ansetzen! Sei es, daß ein wisssenschaftlich tragfähiger Konsens über die Grundlagen erreicht ist, sei es, daß ein Streit mit dem Gegner nicht mehr lohot. Die Zeit der Beschimpfungen - bornierter Krümelsucher hier, spekulativer Sozialphilosoph dort - ist vorüber, und jeder geht seiner Wege.

Die Entwicklung ist aber über diesen Zustand schweigsamer Koexistenz hinausgewachsen. Die Systemtheoretiker machen wieder mobil. Sie haben unterdes das Arsenal ihrer Argumente, so scheint es, beträchtlich verstärkt. In den sechziger und siebziger Jahren bedienten sie sich noch aus den Beständen der Physik und der Kybernetik. Eine wichtige Fundgrube war der zweite Hauptsatz der Thermodynamik. Er bildet im übrigen auch heute noch das Herzstück der Theorie. Aus ihm hatcen schon zuvor einige Theologen - im Hinweis auf die mir der Encropie behauptete Endlichkeit der Welt - einen modernen Gortesbeweis abzuleiten versucht. Die Frage, die auch Soziologen zu denken gab, war, wie sich Ordnung herstellt und wie beständige Selbstorganisation der Materie bzw. der Gesellschaft möglich ist.

Aus dieser Welr also stammen, man nehme einmal sein altes Physikbuch wieder zur Hand, die Begriffe, mit denen rechussoziologische Texte heute ihre Leser auf Distanz halten: Entropie, Negentropie, Dissipation, Reversibilitäc, Irreversibilitäc usw. Die sechziger Jahre bracbren zugleich die beschauliche Phase des soutpue « und sinpur" - jene Zeit also, die uns mit den Begriffen der Kybemetik antreundere: Steuerung, Regelung, Rückkopplung, Homöoscase usw., aber auch die Zeit, die wenig spärer die "Reduktion von Komplexität ", die ursprünglich aus der Kybernetik W. Ross Ashbys' stammte, zum festen Bestandteil juristischer Dissertationen und zum gesellschaftlichen Ereignis auf den Soirées der Gabriele Henkel machte.

Für diese Verwendungsmöglichkeiten werden die Zeiten nun härter. Die systemtheoretische Rechtssoziologie rückt mit Beginn der achrziger Jahre mit biochemischer Terminologie vor. Die Ausrüstung stamme nicht mehr aus den bolzgetäfelten Experimentierstuben des 19.Jahrhunderts, sondern vorzugstveise aus dem von

\footnotetext{
Einführung in die Kybernetik. Frankfure/M. 1976 (englische Eestausgabe 1956). Zum sozialwissenschaftlichen Gebrauch dieses schließlich zum Topos (in der Übersezzung von: Gemeinplazz) inflacıonicren Begriffs vgl. das Vorwort von Jorg Adrian Huber (S. 7-10).
} 
Heinz von Foerster geleiteten Biological Computer Laboratory des Depariment of Electrical Engineering der Universität Illinois.' Die neue Begriffsarmadà segelt hinter dem Flaggschiff "Auropoiesis «, hat in der Rechussoziologie», der Rechestheoriet und neuerdings auch "Rechespolitologie ${ }^{5}$ schon nahezu jedes Thema in Reichweite und nimmt nun Kurs auf die Rechrsdogmatik ${ }^{6}$. Die damit gestelltc Aufgabe verdeutlicht Karl-Heinz Ladeur:

${ }_{n}$ Eine Dogmarik, die nicht mehr an individuellen Ungewißheiten unà dementsprechend cinem parametrisch bestimmten, sondern einem nicht-linearen, dynamischen, auf pluralen Netzwerken aufbauenden Gleichgewichısmodell orientiert ist, müßıe ihrerscits eine neue nich1individueilc plurale Merhode der Kompatibilisicrung und Äquilibrierung von organisationalen Handlungsnetziverken entwickeln.a?

Hier gibe es also viel zu cun. Um aber vorerst auf die Ausgangsfrage nach den Erkenntnisquellen dieser Rechtssoziologie zurückzukommen: Scheint sie nicht durch die vorherstehenden Hinweise ausreichend beantworetet? Die sozialwissenschaftliche Systemtheorie bexieht ihr Wissen offenbar in erheblichem Umfang von den Naturwissenschaften und der Mathematik, an deren Autorität man - jedenfalls im wissenschaftlichen Allıagsgeschäf - nichr zweifeln kann, olne sich zu überheben. Aber nacürlich stellt sich die Frage ganz anders, nämlich: Wie gelingt der Rechtstheorie der spontan kühn anmutende Bogenschlag von einer biologischen Informationstheorie zur Abwägungsproblematik des $₫:$ VI BBauG $?^{8}$ Mit welchen Mitteln spinnen Rechrssoziologen den roten Faden von óer Autopoiesis, einer biologischen Kognitionstheorie (dazu später), zum Steverungsversagen des Gesetzgebers im Sozialrechr?9 Auf welchem Pfade schließlich dringen Rechtspolitologen von der kybernetisch-biologischen Deutung des Hierarchie- und Emergenzproblems zum Mahnbescheid nach $\$ 688$ ff. ZPO vor? ${ }^{10}$ Natürlich gehc es in diesen Fällen nicht um den Anspruch einer systemtheoretischen Hilfeleistung für dogmatische Entscheidungsprobleme. Die Aucoren wollen mit den genannten rechtichen Bezügen jeweils beispielhaft Strukturprobleme des modernen Rechtssystems verdeutlichen. Um eine Vorstellung davon zu geben, wie die Formulierung derarciger Zusammenhänge überhaupt aussehen kann, sollen die zuletzt genannten Autoren etwas ausführlicher zu Wort kommen.

Im Anschluß an das bei Franz M. Wuketis: Biologische Erkenntnis," unter anderem

2 Vgl. Peter M. Hejl: Soziawissenschaft als Theone selbstefercneseller Systeme. FrankfurtNew York 1982, S. 191 .

3 Vgl. die laufenden Nummern der *Zeıtschrír für Rechussoziologıen.

$4 \mathrm{Vgl}$. die baufanden Nummern der Zeitschrife $\times$ Rechiseheorie $\alpha$.

5 Axcl Görlitz, Rudiger Vorgt: Rechispolitologie - Eine Einführung. Opladen 1985, S. 45 iff.

6 Karl-Heinz, Ladeur: Perspckuven eincr pastomodemen Recheshooric - Zur Ausesnandersetzung mie N. Luhmanns Konzepe der "Einhert des Rechissystems $\alpha$. In: Rechistheone 4/1983, S. $383 \mathrm{ff}$.

7 Karl-Heinz Ladcur, a. a. O., S. 426.

8 Karl-Heidz Ladeur: ^Abwägung $\alpha$ - Ein neues Paradigma des Verwaliungsreches. Frankfurz/New York 1984, S. 219 : $D i ß ß$ durch Güterabwagung die Ambigutat der Intcrdependenzvcrhälinisse zwischen Rechrssystem und 2.B. okonomischem Sysem crhöht wird, bedeutet noch kene Funkuonsschwàchung. Hochkomplexe Systeme sınd in der Lage, auch weniger ddirekte Austauschbeziehungen und Funktionsabst)mmungen zu verkralicn, indem sie neue intcrmadiäre Substrukturen ausbilden (t): 50 können z. B. die Abwägungstopor eın erheblich höheres $M a B$ an varıblen, exazächlichen Informatıonen aufnehmen ('soxiale Bedürfmissec), vgl, z, B. $\$$ I Abs. 6 BBauG, zugleich wird dabei eine Vorsirukeurcrungsleıstung des admintstrativen Sysıems vorausgescizt, die die gestergerie Komplexisät durch Anschlußmöglichkeıten für gericheliche Entscheidungen bearbcutbar macht, «

(+) Ladeur verneist hier auf: H. Aclan, L'organistion biologıque et la thíoric de l'informacion, Paris 1972. insbes. S. 261; M.R.Gardner/W. R. Ashby, Connectancc of Large Dynamic (Cybernetic) Systems. Cnescal Value's for Stabiliey, in: Narure Vol. 228 (1970) 784.

9 Gunther Teubner, Helmut Willke: Kontexi und Autonomic: Gesellschaftliche Selbststeverung durch

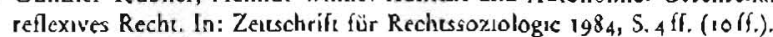

10 Axel Gorliz, Rüdiger Voigr: Rechespolitologie, z. a. O., S. g8.

1r Franz M. Wukets: Biologische Erkenntnis - Grundlagen und Probleme. Stutigan 1983. 
behandelte Problem unterschiedlicher Emergenzniveaus führen Axel Görlitz und Rüdiger Voigt'2 aus:

"Es liegt aul der Hand, daß es außerordentlich schwierig ist, Wirkungskreisläufe auf und zwischen den Hierarchiestufen intcrsubjektiv-transmissibel zu rekonstruieren. Immerhin weisı der - poinciert gesagr - Anschlußzwang auf die Verlaufspotentiale. Eine willkürliche Anschlußhandlung an einen Mahnbescheid wie Herstellung eines Papierheims statr Einlegung eines Widerspruchs licgt insofern im Verlaufspotential eines Mahnverfahrens, als Volistrekkungsbescheid ergeht. Handlungen und Handlungssysteme sind also selekriv relationier, so daß Verlaufswahrscheinlichkeiten entscehen. Hinzu kommu daß die Sclekrivität der Relazionierung von dem Emergenzniveau her erschließbar erscheint. Beispielsweise hal ein Drittschuldner cinem volistreckungsberechtigren Gläubiger binnen zwei Wochen Auskunft zu erteilen, ob und inwiewcir einc Forderung des Schuldners besteht. Versäumt nun der Drirtschuldner die Auskunfítsplichr und rechnet der Gläubiger mit einer negativen Anrwon, darn wärc eine Klage des Gläubigeranwalts auf Auskunftseneilung nicht vom Rechtsstreit, wohl aber vom Mandatsverhàlenis her erklärbar. Auch wenn der Dritschuldner nämlich sogleich die erwartete ncgative Auskunft eneilte, der Rechesstreit also nichts zur Forderungsrealisierung des Gläubigers beitrüge, könnte der Anwalı doch vom insoweit unterlegenen Drittschuldner Gebuhrenersatz verlangen.

Anschlußzwänge zwischen Hierarchieebenen erhöhen die Selekcivität der Relationicrungen weiter. Was aber einer Hierarchieebene noch als komplexe Kombination lateraler Binnenrelationen erscheint, dic symbolisch viruelle Anschlußhandlungen repräsentien, reduzierr sich zwischen Hicrarchiecbencn auf eine adäquate Verknüpfung abgestimmter Außenrclationen. .

Das Problem dieses Texres - einschließlich der Frage, ob er für die hier kritisch betrachtete Diskussion auch als repräsentativ gelten kann - muß hier auf sich beruhen." Das Ausgangsproblem ist nun ausreichend verdeutlichr. Es stellt sich allerdings nicht erst für die neuere Entrvicklung der Rechtssoziologie etc. Die Frage, mir welcher Begründung sie naturowissenschaftlich-mathematische Erkenntnisse zur Erklärung sozialer Phänomene verwende, bedrängc die sozialwissenschaftliche Theorie, seit sie unter dem Dach einer Allgemeinen Systemtheoriè" - übrigens unter dem Zuspruch eines ihrer Gründer, des Biologen Ludwig von Bertalanffy ${ }^{14}$ Uncerschiupf sucht. Das Thema stellt sich heute neu, weil mit der wisscnschafclichen Konzeption, für die der Begriff "Autopoiesis steht, ein Anspruch angemeldet wird, der auf niches weniger als auf eine grundsätzliche Revision vertrauter wissenschaftstheoretischer Posicionen geht.

Die Ausgangsfrage nach den Quellen der systemtheoretischen Rechtssoziologie läßt sich auf zwei Ebenen verfolgen. Einmal ist damic das Problem angesprochen, inwieweit sich theoretische Konzeptionen, die vor allem in nacurwissenschaftichmathemarischen Disziplinen entwickelt sind, in einem nkontrollierten Prozeß der Abstraktion «" zu einer "Allgemeinen Systemtheorie weiterentwickeln lassen. Kann die systemtheorerische Rechtssoziologie sich schon als eiren Teil einer entsprechenden Einheitswissenschaft betrachten? Oder lebt sie in ihrem Zugriff auf naturwissenschaftliche Erkenntnisse von Analogiebehauprungen, bewegt sie sich also in mehr oder minder anschaulichen Gleichnissen und suggestiven Metaphern, die auf spontane Plausibilitätserlebnisse bei den Lesern setzen?

Die sonst so mitteilsame systemtheoretische Rechrssoziologie wird in diesem Punkt ausgesprochen einsilbig. Gegen entsprechende Anfragen der Kritiker, die hier naheliegend den Ansatzpunkt sehen, die ganze Konstruktion aus den Angeln zu

12 Axel Görlitz, Rúdigcr Voıgt: Rechrspolirologat, 3. a. O., S. $\$ \delta$.

13 Eine Bemerkung ser aber schon jeizl gestaiter. Ich haltc dic Besprechung dieses Buches durch Theo Raseliorn in der Zeitschrift für Rechtssozsologıc 1/1986, S. 91 ff., für cin Muster an desinformierender Rezensionspolivik.

14 Ludwig v. Bertalanffy: General Sysiems Theory. 2. Aufl. New York 1969.

is Petcr M. Hejl: Dic Theonc autopoictsscher Systeme: Perspekwen für dic soziologasche Systemeheone. In: Rechistheorte $1982,5,45$ ff. (49) 
heben, ist sie von auffallender Harchörigkeit. Solange in der rechtssoziologischen

Diskussion einzelne Autoren nicht einmal einen Hinweis aut die allgemeine Diskussion dieses Problems' ${ }^{16}$ geben, werden sie sich beständig mit dem Verdacht herumschlagen müssen, daß uncer dem Schein nacurwissenschaftlicher Präzision sich eine mehr oder minder ausgeprägte Beliebigkeit der Argumencation verbirgt, die lediglich von der ausgeliehenen wissenschaftlichen Autorität sog. exakter Wissenschaften lebc. Die im Stande einer derartigen methodologischen Unschuld gezeugten Systeme gehorchen ihren Schöpfern auf das Wort: Sie tun das, was man von ihnen verlangr.

Die damit verbundene wissenschaftscheoretische Erörterung, die in grundsätzliche Probleme sozialwissenschafilicher Modellbildung führt, muß einer gesonderten Behandlung vorbehalten bleiben.

Die Frage nach der Herkunfr systemcheoretischen Wissens in der Rechtssoziologie soll hier auf einer anderen Ebene verfolgt werden. Lassen sich Umstände benennen, unter denen neue Rechtskonzeptionen generiert werden, welche Faktoren leiten die Karriere eines neuen Begriffs, z. B. des der Autopoiesis, auf welche Probleme des Rechts reagiert der Wandel einer Theorie?

\section{Das Recht: Vom Herrschaftsinstrument zur still wachsenden Kraft im Schatten der Kühleürme?}

\section{2.r. „Offenea Systeme: Das Recht als Steuerungsmittel}

Man kann die Entwicklung der systemtheoretischen Rechessoziologie der lezzten dreißig Jahre auf wenigen Seiten auch nicht in Stichworten wiedergeben. Man kann aber in Stichworten dic Probleme dieser Theorie und ihre Entwicklung bezeichnen, die sich in diesern Zeitraum vollzog. Ao den Anfang gehört dabei die Erkenntnis, daß Theorien mit Bezug auf die Gesellschaft und ihr Recht nur dann eine Durchsetzungschance haben, wenn sie auf ein entsprechend aufnahmebereires Problembewußssein treffen. Ohne diese Voraussetzung blcibc der originellste Theoretiker mit seinem Text allein, mag er die Verhälmisse noch so gründlich durchschaur haben.

Das Problem der zweiten Hälfte der sechziger Jahre war die Frage nach der Möglichkeit von politischer Planung. In rechusstaatlich verfaßten Systemen ist dies primär ein Problem der planvollen Veränderung durch Rechc. Die hausbackene Rechtsphilosophie und Rechtstheorie der fünfziger Jahre hatre - von Ausnahmen abgesehen'7 - den Anschluß an die juristische Gundlagenforschung des ersten Drittels dieses Jahrhunderts verloren. Mit ihnen war insoweit kein Staat zu machen. Für den notwendigen Neuanfang war man also auf Importe angewiesen. Diese besorgte gewissemaßen im Alleinvertrieb der vormalige Oberregierungsrac Niklas Luhmann aus Hannover. Seine Schriften ${ }^{18}$ verarbeiteten den amerikanischen Struk-

I6 Peter M. Hejl: Sozıalwissenschaft als Theorıe seibstreferentieller Systeme. Frankfurn New York 1982, insbes. S. 39 ff. mit werteren Nachweisen. Die Diskussion ist U. a. aufgenommen worden von Huber Rottleuthner: Biologische Melaphern im Rechesdenken. Europian University Insutute Florence, Conference Materizls: Autopoicsis in Law and Socisty, Dec. 1985,

17 Z.B. Josef Esser: Grundsatz und Norm in der nehecrlichen Fortbildung des Privatrechts, Tübingen 1956.

19 Für die theoretusehe Verwalnung 5w/ssensehaft gegenuber der betulichen *Verwaliungslehre* fener Zait schon zu Beginn des Jahrzehnis auf konkurrenzlosem Niveau: Niklas Luhmannn: Kann die Verwalcung ornschaflich handeln? Verwaltungsarchiv 11 ( 1960$)$, S.97 fil. Sodann für die Recbissoziologre bedeutsam werdend: derselbe: Lob der Rouene. Verwaliungsarchiv ss (1964), S. Iff.; Offentlichrechtliche Entschadigung - rechespoliusch betracheet, Berlio 1965; Grundrechte als Insutunion, Berlin 1965; Recht und Automation in der offentlichen Verwaltung, Berlin 1966. 
tur-Funktionalismus - tonangebend insoweit: Talcott Parsons - auf Anhieb derart originell und theorerisch ambicioniert, $\mathrm{da}$ ß die juriscische Zunft, über deren incellektuelle Kapaziär man ja schon immer meint, die billigsten Possen reißen zu müssen, sie fürs erste gar nichr zur Kennenis nahm. Das änderre sich, als man sich gegen Ende des Jahnehnts verstärkt nach einer Gesellschafts- und Rechtstheorie umschaute, die die komplexen Verhältnisse des Interventionsstaares im Zusammenhang darstellen konnte, und dabei mit einer gervissen $Z$ wangsläufigkeit auf die funktionalstrukcurelle Theorie Luhmanns stieß. Man konnte sie in dieser Zeit, wenn man wollte, noch als Theorie soffener Systeme* lesen und als theoretischen Rahmen einer rechrsstaatlichen Planungskonzeption benutzen."

Das kybernetisclue Konzept offener Systeme, die im Unterschied zu den geschlossenen Systemen Marerie mit ihrer Umgebung austauschen, ist in den naturwissenschaftlich-mathematischen Disziplinen entwickelt worden, die sich vor allem mit der Weiterentwicklung der klassischen Thermodynamik beschäftigten, deren Gegenstände ursprünglich geschlossene Systeme der Physik und Chemie waren. Für die Sozialwissenschaften wurde diese Theorie alsbald deswegen interessant, weil die mit dem Konzept verbundenen neuen Denkmethoden eine Chance zu bieren schienen, diz gesellschafclichen Strukturen und Prozesse wesentlich differenzierter und vor allem systematischer zu deuten, als dies mic den Kategorien der klassischen Soziologie möglich war. ${ }^{20}$ In Sonderheit das kybernerische Vokabular - Steuerung, Selbstregulierung, Regelkreis, Blockschaltprinzip usw. - nährte die Hoffnung auf ein sozialwissenschaftliches Planungsmodell, das zudem universale Anwendbarkeir versprach."

In dem hier ausschließlich interessierenden juriscischen Bereich ließ sich das Recht mühelos unter das System/Umwelt- bzrv. Input/Output-Schema subsumieren. Alle herkömmlichen Probleme einschließlich dem der Klassenjustiz ließen sich ins Systemtheoretische übersetzen. ${ }^{22}$ Die Konflikte in der gesellschaftlichen Umwelt des Systems werden durch Rechisnormen, die als Transformationsregeln fungieren, in die Form rechtlich entscheidbarer Probleme gebracht. Der rechrliche Tarbestand, die Wenn-Komponente eines Konditionalprogramms - die Terminologie ist mit der Luhmann-Rezeption populär geworden -, filtert die entscheidungserheblichen Informationen, während die Rechtsfolgennormierung das Entscheidungsergebnis programmiert. Das Konditionalprogramm repräsentiert mirhin das klassische Vollzugsmodell des regelgebundenen Rechrsstaats. Eher zuf die Sieuerungsbedürfnisse der politischen Planung hingegen ist die zukunftsoffene Zweckprogrammierung zugeschnitten. Diese Programmform knüpft nicht an die Problemdefinition an, wie sie sich in einem rechtlichen Tarbestand in mehr oder minder konkreten Rechtsbegriffen ausdrückt. Sie definiert vielmehr ein Ziel, das durch rechtliches Handeln erreicht werden soli. Nur solche Mirtel sind zugelassen, die auf die Erreichung dieses

19 Hixerzu vor allem folgende Arbent'n Niklas Luhmanns: Policische Planung (1966); Gesellschafuliche und poliusche Bedingungen des Rechtsstaats (1967); Reform des oflendichen Dienstes: Zum Problem ihrer Probleme (1971). Allesame an: Niklas Luhmann: Poliusche Planung. 1. Aufl. Opladen 1971.

20. Eine gut lesbare Einführung in dic sozialwisscnschafeliche Systemtheone gibe Srefan Jensen: Syseemtheorie, Suusgar, Berlin, Koln, Manz 1981.

21 In den sozıalisuschen Srancen wurde der Plan cbenso als "kybemetische Kategone* frühzeiug crkannt, wie mit dem Konzept selbstregulierender Mechanismen dic freic Konkurrenz markiwustschaftlicher Prozessc erfaße werden konnte. Vgl. hierzu Georg Klaus: Kybcrnctik und Gesellschatt, 2. Aufl. Berlin $1965,5.208 f f$.

22 Vgl. hierzu dic Übersichı be Hubert Rouleuthner: Ruchierliches Handeln - Zur Kreik der juristuschen Dogmarik. Frankfurv'M. 1973, S.142If.; ferner die Diskussion bei Thomas Gawron und Rudolf Schäfer: Justiz und organisterte Inecressen in der BRD. In: Pecer Gral von Kielmannsegg (Hrsg.): Legiumationsprobleme poliuscher Systeme. Sonderhefe $7 / 1976$ der Politischen Viercljahressehrift. Opladen 1976, 5.2171f 
vorgeschriebenen Zwecks - etwa Sicherung der Energieversorgung oder Schutz der Natur - bezogen sind. ${ }^{23}$ In beiden Fällen findet die staatliche Steuerung indes ihre Grenze an der arelativen Autonomie " des organisierten Rechtssystems. Der Jurist denkr hicr spontan an unbestimmte Rechrsbegriffe, Ermessen, Interprezationsspielräume und vor allen Dingen an die Rechtsdogmatik. Letztere ist als "Juristenrecht“ ein genuin systemeigenes Gebilde. Die Dogmatik ist ein Bestand an systemintern erarbeiteten Regeln, Standards, Topoi, anerkannten Konscruktionen, herrschenden Meinungen - kurz: jenes zählebige Gebilde, das durch gezielte Eingriffe von außen kaum veränderbar ist. Der Geserzgeber mag es noch so drücken und kneten wollen, über kurz oder lang wird es wieder seine eigene - nicht norwendig alte - Form annehmen. Vor dieser Eigenschaft mußte schon mancher Reformer resignieren.

Diese Möglichkeit der Selbstprogrammierung ist zugleich Voraussetzung für ein erfolgreiches Lernen des organisierten Rechtssystems, das kritische Informationen aus der Außenwelt in entsprechenden Revisionen seiner internen Strukcuren verarbeiten und sich damit an eine veränderte Umivelt anpassen kann. Außensteuerung und Selbstregulierung schließen sich also nicht aus.

Auf diese handliche Formel etwa wurde die Komplexität der Theorie Luhmanns im Laufe ihrer Rezeption für die Bedürnnisse der Praxis reduziert. Sie tat der Theoric allerdings schon in der Form, in der sie sich in den sechziger Jahren präsentierte, etwas Gewalt an. Denn schon frühzeitig bemühte sich Luhmann, dem dieser Systemauffassung zugrundeliegenden Zweck-Mittel-Schema, das auf einer linearen Verketrung von Ursache und Wirkung beruht, den Gedanken des Bezugs des Systems aus sich selbst ("Selbstreferencialicäta) entgegenzusetzen. ${ }^{24}$ Aber in einer auf Planung, Steverung, Regelung usw. fixierten Zeit konnte dieser Gedanke vorerst keine Breitenwirksamkeit gewinnen. Das änderte sich erst, als mit dem Zerfall sozialliberaler Planungshoffnungen eine planungskritische Theorie gefragt war. Als dieser Gedanke in Bremen angekommen war, ging die Umscellung von Planung auf Evolucion ailerdings um so schneller. Dazu das nächste Kapitel.

\subsection{Autoporests - Eine Theorie zum Selbermachen? Zur Herkunft und Bedeutung des Begriffs}

Andere Zeiten fordern andere Theorien. Die Zeiten der großen Perspektiven und der politischen Planung sind vorbei. Erst reche die der sozialwissenschaftlichen Politikberatung. ${ }^{2}$ Helmuc Schmidt noch suchte im persönlichen Gespräch mir Sir Karl Popper Zuspruch und Rat. Sein Nachfolger findet beides bei Hänschen Rosenthal von Dalli-Dajli.

Keiner weiß mehr so rechr, wie es weitergeht. In solchen Stimmungen, sagt man, wächst die Bereitschaft für das Okkulte. Wenn in der Gesellschaft wieder Jungfrauen schweben und Tische rücken, will die Wissenschaft nicht zur̈̈ckstehen: Bücher schreiben sich wie von selbst, ${ }^{26}$ Endlos-Texte werden aus dem Ärmel

2) Niklas Luhmann: Reche und Automauon, a. a. O., S. 35 ff.

24 Schon zu Beginn der 6ccr Jahre begnnt Lubmann an den Kausalitätsvorstellungen in der Systemtheorie beharrlich zo sägen. Vgl. z. B. dic Kntik cines kausalwissenschaftlichen Funktionsbegrifís in Niklas Luhmanr: Funkuon und Kausadirz̈t. Kölner Zesuschrift für Sozıologıe und Sozıalpsychologie 14 (1962), S.6riff.

25 Deren traunges Schicksal hat Hanncs Frednch: Scaacliche Verwaliung und Wissenschaft. Frankfurr/M. 1970, schon fur das Ende der 6oer Jahre eindrucksvoll dokumentuer. Wolfgang Bruder: Sozjal wissensebafien und Policikberatung. Opladen 1980, konnec eun jahrzebur spater keine Besserung der Situation melden.

26 Auf diese auroporeusche Weise ist Niklas Luhmanns: Sozıale Systeme. Frankfurd/M. 1984, nach Auskunft des Scheu-Autors, der sich lediglich Arrangerprobleme vorbehaten hat, entstanden (S. 1 1). 
geschürcelt. Manche Kleinkünstler - einige kennen wir nun schon - können mir einem Tremolo schreiben, daß Brillengläser zerspringen, und unter der Zauberformel »Auropoiesis» weht der rotgesagte Geist der juriscischen Autonomie wieder aus den Zylindern.

Der Begriff entscammt dem Griechischen. "Autos" heißt "selbst $\propto$ und "poiein nicht nur ndichten « - so die noch h. M. -, sondern auch smachen «. Autopoierisch bedeutet also (sich) selbst machend. Viele müssen das zu wördich genommen haben. Anders ist die theoretische Heimwerkerbewegung, die dieser Begriff auslöste, kaum zu erklären. Whe eine außer Kontrolle geratene Zellkultur wuchert er durch die akademischen Schreibstuben. Das Bild hat insofern seine Berechrigung, als der Begriff aus der Biologie stammt. Wie läßr sich die Organisacion lebender Systeme erklären? Das fragten sich u.a. đie Mitarbeirer des schon erwähnten Heinz v. Foerster, die beiden chilenischen Neurophysiologen Humberto R. Maturana ${ }^{27}$ und Francisco J. Varela ${ }^{28}$. Eine derarcig elementar ansetzende Frage nach den Möglichkeiten von Leben führt alsbald in grundsärzliche Erkennunis(Kognitions)-Probleme. Und genau hier setzc die »Biologie der Kognition $\propto$ an. Das Ausgangsproblem ist an sich nicht neu. Wenn $\bowtie$ Lebewesen $\alpha$ «Leben $\alpha$ beschreiben wollen, müssen sie sich selbst beschreiben. Das erkennende Subjekt - einmal nicht systemtheorerisch gedacht: der Mensch - ist selbst ein Teil der Materie, die er begreifen will. Es läßt sich - für manche sicher etwas ernüchternd - ais bewußsseinsfähiger Zellhaufen beschreiben. Die Materialisten des 18 . und 19. Jahrhunderts fragten sich deshalb, wie sich im Vorgang der Erkenntnis die Materie selbst spiegeln könne. Die Trennung des Betrachters und einer von ihm geschiedenen "Wirklichkeit" war ihnen insoweit nur eine Vorstellungshilfe, weil anders die Realität nicht begreifbar erschien. In der idealisuischen Philosophie drückr sich das reflexive Moment der Erkenntnis in dem prinzipiell unabschließbaren Regreß aus, daß "das Erkennen des Erkennens eben auch wieder ein Erkennen ist $x .{ }^{29}$ Die in derartigen Fragestellungen enthaltenen Zirkel und Paradoxien durchziehen also die ganze Wissenschaftsgeschichre. In früheren Zeiren wollre man den Kreter, der sagre, er lüge, beharrlich und vergebens überführen. Heute bemühr man sich, aus dem "virtuosen Zirkel $\alpha^{30}$ herauszukommen, wie ein Neurophysiologe sein eigenes Gehien erklären kann, wie eine Sprache möglich sei, die "Sprachstrukturen (auch ihre eigenen) zu beschreiben gestatreta, und wie ein System vorzustellen sei, xdas sich reproduziert und dabei auch die Anleitung für seine eigene Reproduktion kopiert und weitergibte. ${ }^{\prime \prime}$

Neu ist nun der Versuch, den Phänomenen des Bewußtseins, des begrifflichen Denkens und der Sprache mit den Mitteln der Naturwissenschaften auf die Spur zu kommen. Die Kognitions-Biologie verläßr sich dabei nicht mehr auf die Denkmittel der traditionellen Philosophie und Wissenschaftstheorie, sondern versucht, ihre

Möglicherwesse hat er emen Schreibautomaten an seinen Zetelkasten angeschlossen; zu dessen forgesclintienen Verselbriandigungstendenzen: Niklas Luhmann: Kommunikatıon mu Zeuelkasten Ein Eriahrungsbenche. In: Horst Baier v. 2 (Hrsg.): Öffencliche Mesnung und soztalcr Wandel - Public Opunion and Soeial Change, Opladen 1981, S. 222 ff.

27 Humberio R. Maturana: Erkennen: Dic Organisauon und Verkörperung von Wirklichketc. 2. Aufl. Braunschweig, Wiesbaden ig8s.

28 Co-Autor von Maturana in: derselbe: Erkennen, a. a. O., S. is 7 ff.: Aucoposese: Die Organisacion lebender Systeme, ihre nähere Bestummung und ein Madel (zus smmen mie R. Vribe); a. a. O., S. 170 lf: : Autopoietische Systeme: Eine Besummung der Icbendigen Organisation (mut ennem - begeisterten Vorwor von Sealford Becr).

29 Friednch Wilhelm Joseph Schelling: Zur Geschichce der neusren Philosophie-Münchencr Vorlesung (1827). Leipzig 1984, S. 101.

10 Gerhard Vollmer: Was können wir wissen? Band 1: Die Natur dur Erkenntnis. Bererige zur Evolucionaren Erkenntnistheoric: mit cinem Gelestwort von Konrad Lorenz, Stuctgar 198, S. 194.

31. Diese und andere Berspicle bei: Vollmer 2, 2, O. 
Ergebnisse empirisch-experimentell zu sichern. wognition ist ein biologisches Phänomen und kann nur als solches verstanden werden. Jede epistemologische Einsicht in den Bereich der Erkennenis setzr dieses Verständnis voraus. (x) ${ }^{22}$

Dieser Anspruch ist insoweit zwar neu-, aber nicht einzigartig. Er ist in den letzten 40 Jahren auf unterschiedlichen Ebenen verfolgt worden. ${ }^{33}$ In Deutschland verbindet sich dic Entwicklung einer biologisch-evolutionären Erkenntnistheorie mit dem Namen Konrad Lorenz, der für seine diesbezüglichen uod anderen Bemühungen den Nobelpreis erhielt. Für den Gedanken, daß unsere Denkmitrel und Erkenntnisstrukturen nicht Produkt eines freischaffenden Geistes, sondern einerseits genetisch bedingt, andererseits stammesgeschichdlich ("phylogenetisch«) erworben, also »ererbte Deakgetwohnheiten" siad, kann man schon Charles Darwin in Anspruch nehmen. ${ }^{34}$

Die ganze Ideenwelt, in die die neuen Probleme eingebetrer sind und die unsere vertraute Vorstellungswelt aufzulösen scheint, hat durch Douglas R. Hofstadter, Gödel, Escher, Bach - Ein Endloses Geflochtenes Band -3s eine gewisse Popularität erhalten. Das verwundert insofern erwas, als das zwar sehr phantasievolle und bilderreiche Buch alles andere als leicht verscändlich ist. Aber vielleicht sollte der Verfasser dieser Zeilen seine beschränkte Kapazität nicht zum Maßsstab des idealen Gesamclesers machen.

Die Neuartigkeit dieser Bemühungen - und das macht sie für die Sozialwissenschaftler inzeressant - lieg? in der Tatsache, daß diese biologische Deutung des Erkenntnisvorgangs sich auch als wangewandte" Systemcheorie lesen läßr. Der Autopoiesis-Gedanke bestimmt das System/Umwelt-Problem auf eigene Weise. Lebende Systeme werden als sich selbst erzeugende Maschinen beschrieben. Sie sind sselbstreferenciell (selbstbezüglich) und "geschlossen «. Mit beiden Begriffen wird eine spezifische Umweltunabliängigkeit, die ja schon im Gedanken der Selbscerzeugung des Systems ausgedrückt ist, behauptet. Natürlich brauchr jedes lebende System eine Uniwelt. Jedes Lebewesen wandelt mit seinem Stoffwechsel Materie um. Mit einem entsprechenden Inpur/Output-Modell, das von einem außerhalb stehenden Betracheer beschrieben wird, läßt sich nach Maturana aber die Organisation von Leben und Bewußtsein nicht erklären. Als biologisches Phänomen ist Wahrnehmung niche mehr Widerspiegelung oder Abbildung einer außerbalb des Beobachters liegenden Realität. Der Begriff der «Geschlossenbeir « von Systemen erhält in der neuen biologischen Systemtheorie eine gegenüber der älteren Kybernetik veränderce Bedeutung. Man kann sich die neue Vorscellung grob im Bild eines in sich gedrehten, an seinen Enden verklebren Papierstreifens veranschaulichen. Das System ist in sich geschlossen. Ein Wesen, das sich mit Scheuklappen nur in dieser geschlossenen Bahn bewegt, macht andere Beobachrungen und Erfahrungen als jemand, der diese Schleife von Außen betrachtec. Ein neuronales Netzwerk - wie es das Gehirn darstellt - ist cin in dieser Weise geschlossenes, nur ungleich komplizierteres System, in das man nur eindringen kann, wenn man es öffner - nur dann ist es kein geschlossenes System mehr. ${ }^{36}$ Die Fremdbeobachtung kann also niemals mit der Selbstbeobachtung eins werden.

$3 z$ Maturasa a.a.O.

3) Vgl. die gur lesbare Darstellung ben: Gerhard Vollmer: Was können wir wissen? Band 1, a. a. O., und Band 2: Die Erkennenus der Narur, Sturtgart 1986; Rupen Riedel: Die Spaltung des Wiltebilders. Biologische Grundlagen des Erklïrens und Versichens, Berlin, Hambung 1985.

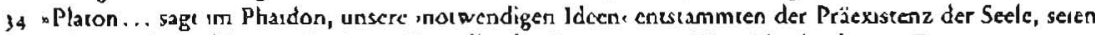
nucht von der Erfahrung abgeletter, lies Affen f́ur Präexistenz. * Diese Tagebuchnouz Darwuss ise ziteert ber: Vollmer, a. a. O., S.XX'Il.

35 s. Aufl. Siutegare 198s (englische Erstiusgabe 1979).

36 Maturana, 2. 2. O., S. 143 . 
„Pnnzıpiell gilt, daß wir - strenggenommen - von einem geschlossenen System niemals Kennenis haben können. Denn um Kennenis von einem System zu haben, müssen wir es beobachien können. Beobachien bedeutec jedoch immer, daß wir mit diesem Sysıem von außenc in Wechselwirkung reten, wodurch dieses notwendigerweise - wegen des reziproken Charakters der Wirkung - in seincr Abgeschlossenheit gestön wird. Bin ich andererseits selbst Tcil des Sysrems, betrachre es also sozusagen von innen, also ohne mich als wahrnehmendes Subjekt, als , lche von dem zu beobachtenden Objekt abzutrennen, so ist sehr wobl Wirklichkeitserfahrung möglich, aber diese Erfahrung kann nichı zu Kennesis und zu Wissen im üblichen, naturwissenschafelich geprägten Sinne gerinnen, da sie nicht objektivicrbar ist. «7

Man kann nach Maturana überhaupe niche mehr sinnvoll die Frage nach dem "Gegenstand der Erkennoris« scellen, denn: „Wir erzeugen... buchstäblich die Welt, in der wir leben, indem wir sie leben.» Oder: "Lebende Sysceme erzeugen also eine spezifische Erscheinungswelt. Ein Beobachter kann immer nur so tun, nals ob er außerhalb der gegcbenen Situation stünde (bzw. von ihr getrennt wäre) ${ }_{\alpha .}{ }^{38}$ Dem voraus liegt die Feststellung,

n... daß Kognivion als ein Plıänomen der Autopoiesc des Erkennenden untcrgeordnet ist, und daB alle kogniciven Zustände als Zustände des Erkennenden durch die Are determiniert sind, in der dieser seine Autopoiese verwirkliche, und nicht durch die Bedingungen der Umwelt, in der ssch dies ereignet. Kognivion isı daher ein prinzipiell subjckcabhängiges Phänomen. ${ }^{30}$

Das folgende Zitat soll eine Vorstellung von der Denkweise des Autors vermitteln:

- Aufgrund der Ar des kogniriven Prozesses und der Funktion der sprachlichen Interakcionen könner wir nichis über das aussagen, was unabhängig von uns ist, und womit wir nicht interagieren können. Dies wïrde eine Beschretbung implizieren, und eine Beschreibung als Verhaltensweise repräsenciert lediglich in Intcraktionen gegebene Relacionen. $D_{a}$ die Logik der Beschreibung die gleiche ist wie die Logik des bescbreibenden Systerns, können wir zwar die epistemologische Norwendigkeit eines Substrats fuir die móglichen Interakcionen behaupren, wir können jedoch dieses Substrat hinsichtlich seiner vom Beobachter unabhängigen Eigenschaften nicht kennzeiclinen. Daraus folgt, daß eine Realicät als eine Welt unabhängiger Gegenstände, über dic wir reden können, norwendigerweise eine Fikrion des rein deskriptiven Bereiches ist...

Die Frage - Was ist der Gegenstand der Erkenmines? wird damit sinnlos. Es gibt keine Gegenstände der Erkenntnis. Wissen heiße fähig sein, in einer individuellen oder sozialen Situation adäquat zu operieren. Wir können über das Substrat, in dem unser kognitives Verhalten gegeben ise, nichı reden, und worüber wir nichı reden können, darüber müssen wir schweigen, wie Wittgenstein bctont hat (vgl. Wittgenstein 1922). Dieses Schweıgen bedeutet jedoch nicht, in Solipsismus oder irgendernc An metaphysischen ldealismus zu verfallen. Es bedeuter, daß wir anerkennen, daß wir als denkende Systeme in eincm Bercich von Besctire:bungen leben, wic bereits Berkeley (1709; 17ro) beiont hat, und daß wir durch Beschreibungen die Komplexität unseres kognitiven Bereiches unbeschränkt vergrößern können. Unser Weltbild und die von uns gestellten Fragen müssen sich daher entsprechend verändern, uso

In diesem längeren Zirat wird man vielleicht vertraute Problemstellungen der philosophischen Tradicion, auf die sich der Text ja auch ausdrücklich berufr, wiedererkennen. Und vermurlich erschließt sich das Moment, das die (sozial-) wissenschaftliche Wele' in Erregung versetzr, erst dann, wenn man sich mit den

37 Hans-Peter Dürs: Über die Norwendigkeit, in offenen Sysemen zu denken - Der Teil und das Ganze. In: Gunter Alener (Hrsg.): Die Wek als offenes System. Eine Kontroverse um das W/erk von Ilya Prigogine. Frankfurr/M. Ig86, S. glf. (11).

38 Maturana, a. a. O., S. 269 und 139

39 Maturana, a.a. O., S. 303

40 Maturana. a. a. O., S. 76 .

41 Daß die Begessterung gerade von Biologen nucht geteilt wurd, sondern sıch auf die Sozıalwissenschaftler beschränkt, ise schon verschiedentlich angemerkt worden. Vol. z. B. Peter M. Hejl: Autoporesis - muß das sejn?. In: Recheshistonsches Journal Bd. S (1986), S. 357 (f. (359). Es ist dies um übrigen erne Verstarkung des Verdaches, daß das Schucksal einer mit naturwissenschafuichen Analogien arbettenden sozıalwissenschafilichen Theone von der Leistungstähıgket ihres Vorbilds werrgehend unabhangıg ist. 
empirisch-biologischen Vorgängen vertraut macht, die ja die Basis dieser Kognirionstheorie bilden. Die Einzelheiren können hier auf sich beruhen. Wir wollen ja an dieser Stelle niche den Geheimnissen des Lebens nachgehen, sondern - wie erinnerlich - dem Geheimnis, aul welche Weise Rechtssoziologen von den Geseczen des Lebens zum Bundesbaugesetz gelangen. Dafür reiche eine ungefähre Ahnung, worum es bei der Konzeption der Autopoiesis ursprünglich geht, vollständig aus. Auf einige allerdings unverzichtbare Einzelheiten kommt der Text im folgenden Kapicel zurück, in dem die sozial wissenschafeliche Rezeption der Theorie behandelt wird.

3. Die rechtssoztologische Einvernabme der Autopoiesis

3.1. Zur Struktur rechtssozialogischer Revolutionen

Immer wenn in der jüngeren Diskussion der Begriff „Paradigmenwechsel a au\}taucht, verweist eine Fußnore roucinemäßig auf das gelesene oder nicht gelesene Buch von Thomas S. Kuhn: Die Struktur wissenschafrlicher Revolutionen.42 Es ist eine wissenschaftssoziologische Arbeit. In ihr beschreibr Kuhn den beschwerlichen und langwierigen Weg, den wissenschaftliche Entdeckungen nehmen mußten, ehe sie sich in der wissenschaftlichen Welt durchserzen konnten. Ein bestimmtes Paradigma, eine nicht nur in der Forschergemeinschaft, sondern möglicherweise auch in der ganzen Gesellschaft eingelebce Wertorientierung, ein allgemein akzeptiertes Problemlösungsmuster oder Theoriekonzept, ein zeitgebundener Denkstil usw. behindern die Durchsetzung eines abweichenden Paradigmas so lange, bis das Wissenschaftssystem in eine Krise gerät, die mit den alten Denkmitteln nicht mehr zu bewälrigen ist. Mancher Entdecker konnte unter diesen Umständen die Früchte seiner Arbeit nicht mehr ernten. Bisweilen mußte er für seine abweichende Meinung mit dem Leben bezahlen. Später erst ermaß die Gesellschaft, auf welche undankbare Weise sie sich von einem großen Geist getrennt hatte. Erst mit dem fälligen Paradigmawechsel vollendet sich eine wissenschaftliche Revolution.

Diese Gefahren sind für Juristen und Rechrssoziologen heute geringer geworden vor allem das Risiko, mit einer grundlegenden Neuerung einfach übersehen zu werden. Sie entledigen sich der Sorge, man könne die von ihnen oder anderen eingeleitere Umwälzung nicht erkennen, dadurch, daß sie den revolutionären Charakter ihrer oder anderer Erkenntnisse schon im Vorwort oder im Verlagsprogramm annoncieren. Es handele sich, so schreiben sie, um ein neues Paradigma bzw, einen Paradigmawechsel. Der Neuigkeitswert eines Gedankens wird also nicht mehr dem Urteil des Lesers überlassen. Wissenschaftliche Revolutionen mereignen « sich heure nichc mehr - weder still noch gar unsichtbar -, wic Kuhn es für frühere Eqochen beschrieb. ${ }^{43}$ Wissenschaftliche Revolutionen werden heute - so scheinc es bisweilen - von den Autoren beschlossen, so wie 1968 , als man in der Mensa wöchentich einmal die Revolution durch das Megaphon für den folgenden Morgen anseczte. Bei so vielen Paradigmenwechsein bleibt es freilich nicht aus, daß man hin und wieder dem Kollegen die Revolucion strcitig machen muß. W Wie dem auch sei: Wir hasten

42 Englisches Onginal 1962, dt. Ubersetzung FrankJuru/M. 1967; tuer zitucen nach der Taschenbuchausgabe, 2. Aufl. 1976.

43 Thomas S. Kuhn, 2.2 O., S. 147 í.

44 Z. B. Kar!-Heinz Ladcur: Perspekeiven, a. a. O., S. 426, Fn. 1 57, ил Zuriekweisung ennes neuen Paradigmas von Dicicr Suhr (vol. Fn. 47). 
vom neuen Abwägungsparadigma" über den Paradigmawechsel in der Juriscischen Merhodenlehre ${ }^{46}$, unter Überfliegen des innovierten Freiheitsparadigmas ${ }^{47}$, mitten in das Katastrophenparadigma ${ }^{48}$. Der Begriff freilich lag schon vorher in Trümmem. ${ }^{49}$ So finder Mao Tse-tungs großer Traum von der permanenten Revolution wenigstens in der westdeutschen Rechtsdogmatik der Post-Moderne ${ }^{j 0}$ seine späte Erfüllung.

Es komme nun vielleicht nicbt mehr ganz unerwartet: Auch wir haben uns jetzt mit einem Paradigmawechsel zu beschäfrigen - jedenfalls nach Auskunft der Beteiligten. Damit er nicht nur bchauptet werden muß, sondern die Umwälzung als solche auch rasch und weichin sichtbar wahrgenommen werden kann, hielt man Ausschau nach einem Begriff, der die Sache traf und zugleich von unverbrauchter Schönheic war. In der biologisch-kybernetischen Sphäre hatte man Effolg. Hier war die „Autopoiesis in kurzer Zeit gleich einem Kometen aufgestiegen. Dessen Schweif harte als einer dec ersten Peter M. Hejl entdeckt. In einer frühen Arbeic ${ }^{51}$ gibr er bereits präzise Hinweise auf Herkunft und weitere Bahnbewegung. Einige Jahre später konnce ihn auch der Laie mit bloßem Auge sehen - Zeit also, daß sich auch Junisten damit beschäfrigten. ${ }^{22}$ Und wie damals über Bethlehem weist uns auch das neuc Himmelsphänomen mit einer Borschaft den Weg: Autopoiesis - das-sich-selbst-Machende bzw. das-von-selbst-Werdende - wurde aisbald als Warnung vor allen vergeblichen Versuchen gedeuret, die Welt planvoll beherrschen zu wolken, und als Mahnung dahin verstanden, wieder den sinneren still wirkenden Kräftena (Friedrich Carl v. Savigny) zu vercrauen, die aus dem Boden der Gesellschaft erwachsen. Oder weniger poecisch:

"Großgesellschaften (sind) nichs mebr durch Rigidicï, sondern nur noch durch Internalisierung und Sponcaneitäh, durch Dezentralisierung, Selbseregulierung und forclaufende Aushandlungsprozesse zusammenzuhalten. "s'

Wie jede gute Botschatt ist auch diese dunkel und mehrdeutig. Auf diesen Fels können viele ihre Kirche bauen. Die Apostel des freien Marktes, die unverdrossen

4) Karl-Heinz Ladeur: •Abwigung“ - eın neues Paradigma des Verwaleungsrechis, FrankfurdM., New York 1984 .

${ }_{46} 6$ Wemer Kraw/erz: Zum Paradigorenwechsel um jursuschen Mechodenstreit. In: Rechestheore, Beihefr I (1979), S. I1) ff.

47 Dieter Suhr: Freihcir durch Geselligkeu, Insumu, Teilhabe, Verfahren und Organisation im sysecmatıschen Raster suncs ncuen Paradigmas. In: Europäisehe Grundrochec-Zeutschrift 1984, S. 529lf.

${ }_{4}^{8} \mathrm{Zu}$ dem $\times 1 \mathrm{~m}$ Anschluß an R. Them, I. Prigogine, J. Petitot und in Óbercinsummung mit G. Günthers Versuch zur Konstrokston einer nachklassuchen, mehrwerugen Logik entwickelien Kataserophenpara-

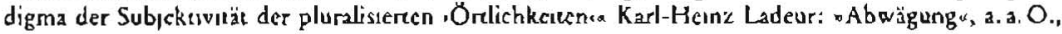
S. 190.

49 Thomas S. Kuhn hat der Demolierung seines Konzepss begreiflicherwerse mir gemischien Gefuhlen zugesehen. Dazu Thomas S. Kuhn: Neue Uberlegungen zum Begriff des Paradigmas. In: ders.: Die Entstehung des Neuen. Frankfurt/M. 1978. S. $189 \mathrm{fF}$. Anders als in der Automobilbranche kennt der Wissenschafusberneb keine Ruckrufakuon für Produkte, dic ihre Benulacr überfordem. Man muB tatenlos zusetien, wis sie rumiert werden.

so Auch dieser Begriff ist postwendend versehlissen worden. Vgl. diesbezüglich die Bemerkung bet Umberio Eco: Posmodcmismus, Ironic und Vergnügen. In: ders.: Nachschrifeen zum Namen der Rose, München, Wien 1984 , S.76 If. In der Postmoderne ist um übrigen alles post: das Denken use postklassisch, die Vemunit postrational, dic Logik postklassisch usw. Die vielen Posiboten lassen künfogen Generauonen kaum noch eme Chance, ihr Zeiralter angemessen zu bezeichnen, sowert niche ene pose-mundiale Epoche diese Frage erledigt.

s1 Peter M. Hejl: Zur Diskrepanz zouschen strukcureller Komplexicäı und traditionalen Darstellungsmıteln der funktonal-strukturedlen Systemcheorıe. In: Franz Macicjewski (Hrsg.), Theoric der Gescllschafe oder Sozialtechnologie. Supplemenr 2. Frankfurt/M. 1974, S. 186 If. (insbes. 194 (I.)

s2 Helmut Rüssmann: Kognıtıve Euhik? - Die Frage nach der W/ahrhecit normativer Sätzc; und Eike Schmidt: Systcmsteuerung durch junsusche Erkenninis. Betdes in: Peter M. Hejl, Wolfram K. Köck u. Gerhard Roch: Wahrnehmung und Kommunikation FrankfurdM, Bum, Las Vegas 1978. S. 279 ff. bzo. 291 if.

3s Walter L.Bühl: Ein neues Paradigma oder enn neuer Myrhos? In: Zertschrift für Politik 1984, S. 333 (336). 
auf die Selbscheilungskräfre eines von staatlichem Dirigismus befreiten freien Spiels der Kräfre setzen, könnten sich hier ebenso angesprochen fühlen wie die Alternativen, die der Allgervalt staatlicher Bürokratien das Modell einer in allen Bereichen strikt dezentralisierten Geselischaft entgegensetzen. Aber beide zeigen sich bislang noch nicht an Autopoiesis interessiert. Die einen haben mit «Monetarismus $\alpha$ einen Leitbegriff, der ihrc Interessen viel plastischer anklingeln läßt, und in den. Wohngemeinschaften hat man sich mit Maturana noch nicht beschäfigt. 's Nicht daß man den nicht peilen würde. Aber es kommt eche zuwenig rüber. Viclleiche wird sich das ändern, nachdem Niklas Luhmann jüngst in der "Frankfurter Allgemeinen Zeitung " den Appell an sie gerichtet hat, sich mit Systemrheorie zu befassen, wobei er keinen Zweifel daran ließ, daß er damir nicht zuletzt seine eigene meinte:

„Dic sneuen sozialen Bewegungen zcigen bisher wenig Neigung, sich mit dieser neuartigen Logik und Kybernetik zu befassen. Das wäre auch nur möglich, wenn sie sich mil Systemtbeorie befreunden würden. Umgekehrt kann sich aber die Systemehcorie mil den sneuen sozialen Bewegungen befassen. a

Letzteres ist mitclerweile geschehen. ${ }^{66}$ Und zwar auf eine Art, die die Alternativen in einer sehr eigenwilligen Weise zur Weirerbeschäfrigung mit det Systemcheorie einlädt:

nSoweit politische Bewegungen, etwa dic ,Grünenı, sich diesern Enrweder/Oder nicht fügen, sondern gleichzents an der Regierung und in der Opposicion operieren möchten, verhalten sie sich obne Verständnis für strukturelle Systembedingungen, und ibre Erfolge können dann nur darin bestehen, Schwierigkeiten zu bereitcn. ${ }^{37}$

Und sodann:

.... man darf Resonanz nicht mit Räsonanz verwechseln. Sonst cntsteht ein Effekı wie mit sgrünen Parteien: Sie haben völlig recht mit ihren Prinzipien, man kann ihnen nur nicht zuhören. s\$ $^{\text {s }}$

Der eine kann nichr zuhören, die anderen wollen nicht lesen - beide aber möchten gehört werden. Schwierige Voraussetzungen für ein Gespräch. Die Kommunikation zwischen Wissenschaft und Polirik wird auf intersystemische Direktheit verzichcen und den Umweg über komplizierte Verrnittlungsagenturen nehmen müssen. Wir rverden gleich sehen, daß damit ein wichtiger Funktionsmodus selbstreferentieller, autopoietischer Systeme angesprochen ist.

\subsection{Selbstreferenz, Selbstorganisation, Geschlossenbett}

Was läßt die systemtheoretische Rechtssoziologiess bei dieser biologischen Theorie der Erkenntnis so aufhorchen? - Neben dem Systembegriffes sind es vor allem die Begriffe Selbstreferenz, Selbstorganisation und Geschlossenheit.

S4 Dafur aber mit dem belgischen Bio-Chemiker llya Pngogunc, vgl. Fn. 37, dessen A rbeıt um das gleiche Problem, närolich das der Selbstonganisation von Marenc, breist.

is Niklas Luhmann: Aleemauve ohne Alemauve - Die Paradoxie der aneuen sozalen Bewagungen *. In: Frankluner Allgemeine Zeitung vorn 2. 7, 2986 . Ob dieses offizıelle Organ der Franklurter Borse der nichrige Platz war? In der taz kónnec der Autor aul mehr Resonanz rechncr.

s6 NikJas Luhmann: Ökologasche Kommurikacion - Kann die moderne Gesellschale sich auf ökologische Gifahrdungen einsiellen? Opiaden 1986. Vgl. dazu gue refenerend Stefan Breuer: Ist Umwelizerstorung überhaupt vermesdbar? In: Merkur $\$ 1$ 1986, S. 681 ff.

37 Niklas Luhmann, a. a. O., S. 171.

s 8 Niklas Luhmann, a. a. O., S. 176.

s9 Liseraturhınweıse zum jüngsten Stand der soxıalwisscrschafelichen Autoposesıs - Rezepuion gibı Gunther Teubncr: Münchhzuscn - Jurisprudenz, in: Rechtshistorısches Journal Bó. s (1986), S. 3 jolf, (3)s f.).

60 Daß gerade der von Maturana/Vanela verwendete Systemjangon dic Theorie für Nichebiologen alurakuv machi, erkennt zurreffend Peter M. Hejl: Autoparesis - muB das sein?. In: Rechishistonsches Journal Bd. s ( 1986$)$, 357 ff. (358f.): - Für sie taucht hier ein offenbar naturwissenschaftlich abgesicheriese neues 
Der Sache nach gehr es um eine veränderte Sicht des Rechtssystems. Man sieht es nun nicht mehr primär unter dem Gedanken seiner planvollen Steuerbarkeir für bestimmte Ziele. Auf diese Aufgabe war die Konzeption des offenen, in bestimmter Weise "fremdreferentiellen ${ }^{\sigma_{1}}$ Systems bezogen, wie es oben vorgestellt wurde. Das Rechtssystem wird also jetzt nicht mehr wie ein Transformator gesehen, wie es das Input/Output-Schema nahegelegt harte: Ein Lebenssachverhalt oder eine bestimmte Aurgabe wird cingegeben, nach bestimmten Regeln transformiert, und als Losung: Ureil, Verwaliungsakt oder eine ähnliche Entscheidung, verläßc es das System. Dieses instrumentelle, an eine Maschine crinnernde Funktionieren gibt es zwar auch noch - und zwar im wörlichen Sinne, wenn man an die zunehmende Auromarisierung bei Gerichten und Behörden denkt. Aber dieser Sonderfall würde als allgemeines Modell des Rechts dessen gesamrgesellschafeliche Funktion verfehlen. Es ignoriert die Autonomie und Eigendynamik des Rechts. Wir hatten gesehen, daß die Rechtssoziologie vor der Entdeckung der Autopoiesis natürlich auch diesen Gesichtspunkt registriert hatte. Aber soweit dies im klassischen System/UmweltModell geschah, war diese Auronomie lediglich Vorausserzung der $\bowtie$ Anpassung« an dic Umwett und das ganze ein Vorgang der Bestandserhaltung des Systems.

Diese Sicht ist mir der neuen Konzeption sozusagen ausgewechsclt worden, wobei die Auropoiesis gewissermaßen den Schlußstein dieser Entwicklung bezeichner. Die Begriffe deuten es an: "Selbstreferenz löst die Außenlenkung ab, "Selbstorganisation « die bloße Anpassung durch Systemlernen, und "Geschlossenheit" verabschiedet die Vorstellung, daß das System/Umwelt-Vcrhältnis als direkter Austauschprozeß zu denken sei. Die Begriffe srehen dabei in einem engen Zusammenhang. Die Rechtssoziologie hat mit dieser Entwicklung den Akzent von Planung auf Evolution verschoben. Diese Begriffe schließen, soweit sie reale Prozesse bezeichnen, sich nicht unbedingt aus. Auch in einern evolutionär gewachscnen Rechtssystem kann geplant werden. Aber sie lassen sich gur für eine Kontrastierung der alten und neuen Vorscellungen verwenden. Planung wird betrieben - und $z$ war durch ein eigens für ihre $Z$ wecke geschaffenes Recht, das insoweit einen rein instrumentellen Charakter hat. Die Evolution hingegen vollzieht sich unbeeinflußbar, sie kennt keinc lenkende und auch keine unsichtbare Hand, die Adam Smith noch über dem Marktgeschehen walten sah. Sie verläuft unkontrollierbar, in spontanen Sprüngen, mit prinzipie!l unerwartbaren Ergebnissen. Sie hat keinen angebbaren Grund und kein berechenbares Ziel. Übersetzt in die wissenschaftliche Sprache: Das klassische Planungsmodell arbeitet mit einem linearen Zweck/Mircel-Schema. Dessen Logik is hierarchischdeduktiv. Sie ist von Kausalitätsvorstellungen geprägt. Das Steuerungskonzept denkt teleologisch im Sinne von zielgerichtet und zielbestimmt. Evolurionäre Prozesse hingegen lassen sich nicht als lineare Ursache-Wirkungsketren beschreiben. Das cvolutionäre Denken ist insoweic akausal. Die komplexe Struktur evolutionärer Prozesse läßt sich däher nicht in der Dualität der klassischen Logik begreifen, sondern erforden eine andere Logik, wic sie manche in dern Konzepi einer mehrwertigen Logik sehen. ${ }^{62}$

Systemmodell zur gefäligen Weaervervendung auf. Ohne daß die geringe Resonanz der Autoposese Jieone bei Biologen zum Warnsignal wirde, wird das Modell nicht nur ubemommen, sondem sverallgemeinert $\alpha$. (359).

6i Dazu Peter M. Heil: Sozralwsssenschalt als Theone selbstrelerenteller Sysisme, FrankfurdM., New York 1982, S. 178 ff.

62 Die Selbstverständlichkeit, mit der diese Vokabeln un der Rechtssoziologte mitunter benutzt werden, läßt leicht vergessen, daß nahezu jeder Begriff in der naturwissenschafelichen Evolutionsforschung für eine Kontroverse steht. Das gilt für den Begriff der Kausalität ebenso we für die Erörterungen, die um den Telos-Begriff kreisen (Entelechie, Teleologae, Teleonomie). Auch die Reicliwerte der klassıschen zweiwerigen Logik wird unterschiedlich eingeschaizt. Vgl. Gerhard Vollmer, z. a O., Bd. 1, S. s7ff: 
In der deutschen Diskussion war es Niklas Luhmann, der 1970 die "Emeuerung des Evolutionsgedankens $\alpha^{6_{3}}$ in der Weise betrieb, daß er die „neuc Welle des Evolutionismus ${ }^{64}$, die er aus Amerika heranrollen sah, auf seine Mühlen lenkre und aul diese Weise eine Evolutionstheorie erzeugte, die weniger als Wiederaufnahme, sondern eher als cin Neuanfang und in diesem Sinne nicht nur als originell, sondern auch als originär gerverter werden muß. Zehn Jahre später schwollen die bis dahin vereinzelten Rezeptionsansätze dieser Konzeprion unvermittelc zu einer Bewegung an, die eindeutig auf die vermeindliche oder tatsächliche Krise des Interventionsstaates reagierte. Es war die Zeit, als der Hamburger Bürgermeister Klose seine Stadt für unregierbar erklärtc, Politologenkongresse sich eilfertig dieses Eingeständnisses annahmen, ${ }^{63}$ und später sogar Juristen den Staat enczauberten, ${ }^{66}$ kurz: als die Hoffnungen auf den allmächtigen Planungsstaat der Klage über die Unregierbarkeit des Landes wichen. Einer der wenigen, die sich nach der Hcrkunft der Wellc fragten, auf der die Bewegung seither reitet, war Gunther Teubner:

-W/arum ist in der Rechtstheorie heute Evolutionismus wieder en vogue? Welche Motive steuern das neuerliche Interesse an soziologischen Entwicklungsmodellen des Rechts? Vermutlich finden die im einzelnen sehr disparaten Enrwicklungsmodelle ihre Einheit in einem gemeinsamen Problembezug. Sie alle suchen in evolutionärer Perspektive Antworten auf die eine Frage, nämlich wie das Recht auf die Krise seiner spezifischen modernen Rationaliuä - der "formalen « Rationalizät - reagıen, warum diese Krise entsıanden ist, welche Auswirkungen sie hat und ob eine Krisembewältigung - oder auch nur ein K risenmanagement - in Sicht ist. ${ }^{67}$

Das Theoriegebäude wird also renoviert. Der Muscerplan liegs vor, und man gehr mit Eifer zu Werke. „Theoriebautechnisch gesehea - gibu Werner Krawietz ${ }^{68}$ gewichtig die Parole aus, als längst alles am Umbauen ist und der Architekt sich schon wieder anderen Projekten zuwendet ${ }^{69}$-, wTheoriebautechnisch gesehen, geht es heute um einen Umbau der Theorie adapriver Systeme zu einer Theorie selbstreferentieller Systeme. Wer das niche begreife, ignoriere »die neuesten Encwicklungen in der Systemtheorie - nämlich von bloßer Anpassung zu autopoiecischer Evolution!

Man kann das ganze Unternchmen vielleicht etwas unbeschwerter begleiten, wenn man sich vorab klarmacht, daß die Umscellung von Planung und Anpassung aul Evolution und Selbstorganisation des Reches den Gegenstand, um den es eigentlich geht, nicht verändern, sondern nur anders beschreiben und bewerten kann. Es ist das Problem der juristischen Autonomie. Die Frage also, ob das Recht wsein eigenes

-Dic Evolutionstheorie, wie sie beuce vor uns stcht, sst keine voll ausgearbeitcee Theorie, sondern cher en Forschungsprogramm" (\$. 11i). Hier gehe es um ene grobe Markienung der Vorstellungen, an denen sich nicht dic Thcorıe Macuranas, wohl aber ihre populärc Rezeption orientucr. Man erkennt die grobe Richtung, die dic Diskussion numme, dic in Einzelhesten natürlich nichr un paralleler Geradlinigkest verläuft.

6) Niklas Lubmann: Evolution des Rechis (1970). In: ders.: Ausdifferenzerung des Rechts, Frankfure/M. 1981, S. 11 ff. (12). Vgl, auch ders.: Rechissozialogic L. Rernbck 1972, S. 132 ff.

6. Niklas Luhmann: Evolucion, 2. 2. O., S.11.

6) Das Therna hal, wie fast jedes gescllschaftliche Thema, eine bis un dic Antikc zurückrcichende Tradition. Vgl. Theodor Schicatr: Einmaligkeıl oder Wiederkehr - historische Dimensionen der heuugen Krise. In: Wilhelm Hennis/Peter Graf Kielmannscgg/Ullich Matz (Hrsg.): Regierbarkcit, Bd. 1, Siungart 1977, S. 22 if.; vgl. auch Karl Ottmar von Areun: Das Problem der Regierbarkeic um Heiligen Romischen Resch. In: Wilhclm Hennis u. a. (Hrsg.): Regierbarkcut, Bd. 2, Stuugar 1979, S. Iff.. Für den vorlicgenden Zusammenhang ist nur der Hinwers auf die politische Konjunkturabhain. grokest der Thematik wesentich.

66 Helmut Willke: Dic Enuxauberung des Sta2tes, Kanigstein 1983.

67 Gunther Teubner: Reflexives Rechi - Enrwicklungsmodelle des Reches in verglachender P(rspekuve. In: Arclsuv für Reches- und Sozialphilosophic 1982, S. 1, fif. (14).

68 Wcrner Krawiciz: Reche und modeme Systemtheoric. In: Rechescheorie, Beiheh 10 (1986), S. 281 if. (291, Fn. 60).

69 Niklas Luhmann, A. ส. O., passim und allerwarts. 
Daseyn (Friedrich Carl v. Savigny) habe. Man wird sehen, ob dieser uralte Zankapfel ${ }^{\circ}$, der für viele schon längst gegessen schien, seinen ideologischen Geruch, der ihm stees anhaftete, verliert, wenn er in die keimfreien Begriffe der Systemtheorie getaucht wird.

\subsection{Uber die Zirkularität in der Evolution des Rechts: Von der Begriffsjurisprudenz (1850) zur Begriffsurusprudenz (1986)}

Zunächst wird sich der an der ganzen. Diskussion nur beiläufig interessierze Leser fragen, in welchem Sinne man in einer Zeit von rechtlicher „Evolution * sprechen kann, in der man täglich von der Normenflut nicht nur in der Zeitung liest, sondern ja auch selbst ständig von ihr betroffen ist. Gestern hatte man sich mit Gurten anzuschnallen, heute muß man seinen Zahnersatz, wenn er nicht nur aus rostfreiem Stahl geferrigt sein soll, selber bezahlen, und morgen wird man, so ist versprochen, weniger Steuern zahlen. In allen Fällen gehorchc man präzise definiernen Rechusbefehlen, denen außer der Tatsache, daß sie zur Strategie eines politischen Überlebenskampfes gehören, nichts Evolutionäres anhaftet. Sie sind positives, gesetztes Recht, das ganz unevolutionär heute so und morgen so ausfallen kann.

Die systemtheoretische Rechtssoziologie stellt diese Form der Recheserzeugung делn auch nicht in Frage. Sie deutet die Gesetzgebung selbst nicht als evolutionären Vorgang - jedenfalls niche in der angeklungenen Bedeutung. Die Gesetzrheir und jederzeirige Änderbarkeit des Rechts gilt ihr vielmehr als das Ergebnis eines insoweit vorerst kaum mehr revidierbaren gesellschaftlichen Prozesses.

Gegen diese Entwicklung hatte sich mit Erfolg das letztc Mal (in der ersten Hälfre des vorigen Jahrhunders) die sog. Historische Rechtsschule unter Führung von Carl Friedrich v. Savigny gestemmt. Sie sah in der wWillkür eines Gesetzgebersu vielerlei Gefahren auf das Recht zukommen - nichr zuletzt auf den Juristenstand, dessen Bedeutung natürlich in dem Maße zurückgehen mußre, in dem ihm seine Verantwortung für die Rechtssetzung durch einen aussch)ießlich nach Zweckmäßigkeitsgesichtspunkten entscheidenden Gesetzgeber abgenommen würde. Man fürchtete sich vor der subalternen Rolle des bloßen Paragraphenanwenders." Aber gegen die drohende Willkürherrschaft des Gesetzgebers wurde narürlich nicht mit berufsständischen Parolen gefochten. Ein politischer Kampf hat mehr Aussicht auf Erfolg, wenn sich das ständische Interesse als Algemeinwohl präsentiert. Das war damals nicht anders als heute. Damals sprach man vom "selbständigen Daseyn « des Rechts, das der Obhut der Juristen nicht entrissen werden dürfe, wenn es nicht schweren Schaden nehmen solle.

Die Summe dieser Ansicht also ist, daß alles Recht aul die Welse entsteht, welche der herrschende, nichı ganz passende Sprachgebrauch als Gewohnheiesrechr bezeichnet, d. h. daß es erst durch Sitte und Volksglaubc, dann durch Jurisprudenz erzeugt wird, überall also durch innere, still wirkende Kräfte, niclst durch die Wrillkür eines Geserzgebers. «" ${ }^{71}$

70 Diese Fruche halse ich ein wenıg hin und her gerolle in: Joachım Nocke: Über die Bestündigkeı des Jurisien als solchen - Ineerdisziplinäre Arbeil der Recheswissenschafe vor dem Ánspruch auf jurisusche Autonome. In: Jurgen Simon (Hrsg.): Regulierungsprobleme im Winschatessysecm - Beitrige eum Markt, Verwalung. Recht. Neurvied u. Damsude 1986, S. 12 fff.

71 Darın sah en halbes Jahehunder späar Max Weber immer noch das maßgebliche Mouv des Juristenstandes für sesnen Iretrechelichen Versuch, dem Geserzgeber rechusschópferische Funktionen abyutrotzen. Vgl. dazu Beatnce Caesar-Wolf. Der deusche Richter am "Kreuzweg awschen Prolessionalisierung und Deprofessionalisterung. In: Stefan Breucr/Hubert Treiber (Hrsg.): Zur Rechissoziologie Mix Webers, Intcrpreution, Krikik, Weiterentwicklung. Opladen 1984, S. 199 if.

72 Friedrich Carl von Savigny: Vom Berul unserer Gesetzgebung und Rechuswissenschaí, Heidelberg 18:4, wiedcrabgedncke in: Thibaut und Savigny - Ihre progcammatischen Schrifien. Mit einer Einfülyyung von Hans Harrenhauer, Munchen 1973, 5. 95 ff. (10g) 
Diese Ansicht hatte allerdings nicht nur ein standespolitisches Moriv, sondern auch einen ernst $z u$ nehmenden theoretischen Hintergrund.73 Dazu sogleich mehr. Zunächst gilt, daß sich für die Historische Rechusschule alles Recht einem stillen Wachstum verdankt, das durch keine unkundige Hand gestört werden soll und das auf diese Weise im Laufe der Geschichte seiner vollendecen Gestalt entgegenstrebt. Es ist ein evolutionärer Prozeß - dies allerdings im klassisch-biologischen Sinne des Begriffs, wie ihn z. B. Darwin benutzte, der übrigens zu seinen bahnbrechenden Ideen ursprünglich nicht durch die Beobachtung von Lurchen, sondern durch die des geschichtlichen Enrwicklungsprozesses des Rechts angeregt worden sein soll.

Der Evolutionsbegriff der gegenwärtigen Systemtheorie hat damit kaum etwas gemein. ${ }^{7-1}$ Mit dem Sozialdarwinismus etwa, der noch heute die gesellschaftliche Entwicklung in Analogie zur organischen Evolution sieht, indem er z.B. die Probleme unserer Zeit auf die Verhausschweinung des Menschen, die Bedeutungslosigkeit seiner natürlichen Feinde - Schlangen, Raubkatzen, Drachen u. ä. - und die zu erfolgreiche Bekämpfung von Seuchen zurückführt,"s hat der systemfunkrionale Evolutionismus im wesentlichen gebrochen. Und auch sonst scheint jeder gedankliche Brückenschlag vom selbständigen Daseyn des Rechts zur Autonomie des autopoietischen Rechts zunächst etwas zwanghaft. ${ }^{76}$ Die Positivität des Rechts, die im Laufe des 19. Jahrhunderts die Niederlage der Historischen Rechrsschule besiegelte, ist ein Grundpfeiler der systemtheoretischen Rechtssoziologie, 77 und von der ganzen quasi-naturrechtlichen Metaphysik, mit der die Historische Rechtsschule ihre Schwachstellen einnebelre, hac der - obenlächlich - konsequent bekenntnisfreie Systemfunktionalismus nichts übriggelassen. Um so mchr wird deshalb die Nachriche überraschen, daß Luhmanns Argumentation auf nichts seniger als auf ein mehr oder weniger explizites Plädoyer für die Begriffsjurisprudenz hinausläuft ${ }^{78}$ jene vielgelästerte und heute selbst von skurrilsten Dogmatikern als Beschimpfung aufgefaßte Rechtslehre, die entgegen der landläufigen Meinung allerdings keine Karikatur, sondern die konsequente Vollendung der Historischen Rechtsschule gewesen ist. ${ }^{79}$

Daß ausgerechnet eine in computergesteuerten Genlaboratorien generierte Theoric, nämlich die Autopoiesis, zur Wiederbelebung einer in den Beständen des 19. Jahrhunders verstaubenden Idee geeignet sein soll, muß auf ungläubiges Staunen

73 Zu den nicht ohne werteres sul emen Nenner zu bringenden philasophischen und polituschen Einflüssen, unter denen die Arbeıt des Schulenhauptes stand: Joachım Rücher: Idealismus, Jurıspradenz und Policik bes Friedrich Carl von Savigny, Ebelsheim 1984

74 In dem als Evolution zulgeiaßten Prozeß der fortschreitenden Diffetenzierung geht es niche um einen ingend wne geartetcin Überlebenskampf, beı dem das eıne dem anderen endgülug zu weichen häute oder durch sonstuge Sclektionsmechanismen ausgemerzt würde. Jencr Vorgang vollzieht sich vielmehr als Steıgerung von Komplextı̈̈t. Sozıale Ausdifferenzierung geschıeht in der Ausbildung von kommuniksuven Zusammenhängen. die aul funktıonsspezifische Sinngehalıe bezogen sind. Bei Kommumikatıonen gilt grundsätrlich, daß cin móglicher Sinngehale nucht dadurch vemıchtet wird, daß die Kommunikatıon èn anderes Thema mie anderen Sinnbezügen wählt.

7) Aul diesen Siand isı dem Vernehmen nach Kanrad Lorenz: Der Abbau des Menschlichen, München 1983, angelangt. Vol. S. 208. Lorenz spriche u. a. von cincr Sacculinisierung der menschlishicn Evolution (S. sa Il.): Der Sacculina Carcun ist cin Krebs, der sıch als Parasıl zul einer Strandkrabbe cun schönes Leben machs, um in der Folge sein nichr mebr benougtes Nervensystem, Auge und Exuremiäten cinzubüßen. Hinwers und Zusammenfassung verdanke ich Walter L. Bühl: Ein neues Paradigma oder eın neuer Myehos?. In: Zenschrifr für Policik 1984, S. 333 If. (334).

76 Vgl. heerzu Sibylle Tonnies: Organısmus und Freiheı - Der Verlust der Subjektıvität un Luhmanns Systemtheone. ln: Hans-Ernst Börtcher: Recht Justız Kritik. Festschnft für Richard Schmid zum S5. Geburestag, Biden-Baden i985. S. 375 ff.

77 Niklas Luhmann: Positıvic̈z des Rechts als Vorausserzung esner modernen Gesellschade. In: ders.: Ausdifferenzerenong des Reches, a. a. O., S. I 13 ff.

i8 Niklas Luhmann: Rechessysecm und Rechesdogmatik, Stutgart U.A. 1974; dors.: Dic Einheil des Rechissystems. In: Rechestheone 1983 , S. 129 if.

79 Weitere Nachweise dazu in meunem Aufsaca (Fn, ;0). 
stoßen. Und in der Tat verweigern die Jünger bej diesem Gang über das Wasser dem Herrn die Gefolgschaft. ${ }^{80}$ Kleinmürig verharren sie am Ufer des Sees. Das Mißgeschick Petri schreckt. Dabei ist jener wagemutige Wandel nichts anderes als eine sich aus der Anlage der Theorie mehr oder minder zwingend ergebende Konsequenz. In dem ausschließlich aus sich selbst heraus lebenden, sich in einer "Genealogie der Begriffe" (Friedrich Puchca) ausschließlich von selbsterzeugten Elementen fortzeugenden, geschlossenen, eigenexistenten System finder das Modell eines aucopoietischen Rechrs seine reinste Enrsprechung. Nachfolgende Zeiten, die in diese Idylle mit politischen Zwecken, rechtsfremden Interessen und soziologisierenden Rechtsfolgeerwägungen herzlos eindrangen, müssen dem autopoietischen Recht als Abirrung vom rechten Pfade vorkommen. Und eben dies wird beklagt. ${ }^{81}$ Sie bringen das System um die Errungenschaft einer Evolution, so wie die Systemtheorie sie versceht: als beständig fortschreitende soziale Differenzierung der Gesellschaft. ${ }^{{ }^{81}}$ Die soziale Differenzierung - Herzstück der funkcional-strukturellen Gesellschafrstheorie - betrifft zunächst den Prozeß der allmählichen Herausbildung eines selbständigen Rechtssystems im Zuge der gesellschaftlichen Entwicklung. Er führt also vom Regiment alter Stammesoberen, die noch viele - nicht alle - Aufgaben, die wir heute als politische, religiöse, rechtliche, erzieherische Funkrionen bezeichnen würden, in sich vereinigıen, zu der Ausbildung mehr oder minder autonomer Funktionssysteme, wie sie das Bild moderner Gesellschaft prägen: Polizik, Recht, Religion, Wircschaft, Erziehung, Wissenschaft usw. Die für unseren Zusammenhang nun wesentliche Einsicht ist die, daß diese Arbeitsteilung, wie sie Emile Durkheim ${ }^{8_{3}}$ beschrieb, nur funktionieren kann, wenn die beteiligten Funktionssysteme wirklich autonom sind. Der Gedanke leuchtet ein. Wenn im Recht die Probleme der Wirtschaft, in der Religion die der Policik und in der Medizin die der Erziehung gelöst würden - kurz: wenn einer die Arbeic des anderen miterledigte, würde das die gesellschaftliche Ausdifferenzierung von Recht, Wirtschaft, Politik usw. um ihre Früchre bringen. Deswegen sind politisierende Prälaten, soziologisierende Juristen und therapierende Pädagogen dieser Sicht verständlicherweise ein Greuel. Sie sind Ausdruck einer schleichenden Entdifferenzierung des Systems. Und eben dieses Argument war ungeachtet mitlaufender Professionsinteressen der ernst zu nehmende theoretische Hintergrund der Historischen Rechtsschule. Die soziale "Sonderung der Thätigkeiten" war schon für Savignys Konzcption ein bedeutsamer Tatbestand. ${ }^{84}$ Und die großen Theoreriker der späteren Begriffsjurisprudenz ${ }^{8 s}$ haben im Hinweis auf diesen Zusammenhang die juristische Autonomie

8o VGl. z B. den im übrigen lesenwerten Rufsalx von Gunther Teubncr: Verrechdichung - Begriffe, Merkmalc, Grenzen, Auswege. In: Hans F. Zacher/Spiros Simstus/Fnedrich Kübler/Klaus Hopt/ Guneher Teubner: Verrechtichung von Wiruchaft, Apbett und sozıaler Solidantät, Baden-Baden 1984 , S. 189 ff. ( 311 f.), der einen 2 war pietölvallen, aber endgülugen Nekrolog auf den Windscheidschen - Jurnsten als solchen formuliert, Jen Luhmann gerade reanimieren möchte, indem er ihm den Geist der Autoposesis einhaucht, ohne daß der Totgeglaube freilich busher außerbalb der Theorie Luhmanns wieder Lebenszeschen von sich gegeben hälte.

8. Niklas Luhmann: Dic Einhcit des Rechissystems, a. a. O.

8: "Rechiskntik us demnach im Kern: Kntik der gesellschátlichen Differenzicrung. So Niklas Luhmann: Die Einhert des Rechtssysecms, 2. 1. O., S.153.

83 Emile Durkheım: Über dic Teilung der sozulen Arbeıt (1893) - engelestet van Niklas Luhmann, Frantefure/M. 1977; vgl. auch Friedrich Jonas: Geschichte der Soziologie. Bd, 2, Reinbek 1977, S. 1 ff.; sebließlich die Beusüge ın: Niklas Luhmann (Hrsg.): Sozıalc Differenzıcnung-Zur Geschichte eıner Idee, Opladen ings.

84 v. Sawgny, a.a. O. (FN 72) S. 104. Zu diesem Zusammenhang vor allem Peter von Oenzen: Die sozıale Funkuon des suatsrechelichen Positivismus (1953). FrankJurdM. 1974, und Walter Wilhelm: Zur jursustischen Methodenlehre des 19. Jahrhunderes, FrankfuruM. 1958.

8s Man lese unter diescm Gesıchispunke eunmal die schönc Schrift von Hugo Preuß: Zur Meshode junstuscher Begriflskonstrukuon. In: Werner Krawietz (Hrsg.): Theorıc und Teclunik der Bcgriffsjurssprudenz, Darmscadi sa76, S. 1 isfl. 
gegen die Anfechtungen einer härter werdenden Merhoden- und Rechtskritik, mit der sich die Freirechtsschule und Interessenjurisprudenz ankündigten, in Schucz zu nehmen versucht.

\subsection{Die Krise des Rechts: Feblende oder gesteuerte Steuerungszentrale?}

Der Zusammenhang von Autopoiesis, Autonomie und Evolution - letztere sowohl in der alten wie in der neuen Bedeutung des Begriffs - ist jetze in Umrissen erkennbar. Worin besteht nach Auffassung der Beteiligten nun die Krise des Rechts, auf die der moderne Evolutionismus offenkundig reagiert?

Stichwortartig läßc sie sich so wiedergeben: Der Glaube an die Möglichkeit, die Gesellschaft bzw. ihre Probleme mit den Mitceln des Rechts hierarchisch steuem zu können, ist dahin. Die Welt ist zu komplex geworden. Die gesellschaftliche Differenzierung ist für eine rechtsförmige, zentralstaatliche Lenkung zu weit fortgeschritten. Die einzelnen Funktionssysteme Recht, Wirtschaft, Erziehung usw. entziehen sich nicht nur einem Primat der Politik - wenn dies jemals bestanden haben soll -, sondern generell jeder gezielten Beeinflussung. Planungen verfehlen unter diesen Umständen zunehmend ihr Ziel und erseichen nicht selten das Gegenteil von dem, was sie erstrebten. Kurz: Der zentral agierende Wohlfahrtsstaat stößz auf seine Grenzen und ist in vieien Bereichen melu oder weniger am Ende. ${ }^{86}$

Was an dieser Ausgangsannahme zutriffe und was nicht, muß hier - diesmal aus Platzgründen - dahinstehen. ${ }^{87}$ Man muß sich nichc erst in die umfängliche Implementationsforschung einarbeiten ${ }^{88}$ und die von ihr belegten "Vollzugsdefizitea staalicher Programme bemühen, um an die Grenze der staatlichen Durchsetzungsmöglichikeiren zu glauben. Jedes $\mathrm{Bad}$ in der Mosel bieret genügend Empirie. Ein Hinweis scheint allerdings in diesem Zusammenhang angebracht. So anonym, wie es sich für eine im Abstrakrionsfieber schreibenden Systemtheorie darstellt, sind die Kräfte nichi, die hier am Wirken sind. Und es ist auch nicht erst ein "Vollzugs wProblem, das die "Zentraldemokratie " ${ }^{89}$ lähmt. Der Befehlsgeber wird vielfach schon an seinen Befehlen gehinderc. Abgesehen von der mittlerweile anschaulich belegten Tarsache, daß man mißliche Geserze, im Steuerrechs etwa, durch ein im Vorzimmer des Ministers zurückgelassenes Kuvert abkaufen kann: Die Industrie droht auf Gesectesankündigungen unverhohlen mit Abwanderung in ein investicionsfreundlicheres Klima. Sie nimmt ungehemmt Einfluß auf Wahlen, indem sie für

86 Fine unter diesen Pramissen theoreusch präzıse argumentıcrende Darstellung findel sıch beı: Niklas Luhmann: Politische Theoric im Wohlfahrussuat, München, When 1981 vgl. auch Helmut Willke: Die Entzauberung des Sizates, a. a.O.

87 Was die empirischen Grundlagen dieser These - eiwa auf dem Gebicı der Wirescbaíslenkung anbelangt, hat Petcr Nahamowite: , Reflexives Rechro: Das unmögliche Ideal eincs post-mitervenuonisuschen Stcuerungskonzeprs, in: Zeitschrif für Rechtssozsologie 1/1985, S. 29 ff. dankenswerterveise eınges wheder zurechtgeruckt. In der Tat ist die empinsch schwachbelcgic und unsowelt unbegrundet gencralisicrende Rede vom Stcucrungsversagen des Staatcs, dic eincn Teil für das Ganze nirnmt, cine der Hauptschwächen sawohl der aul dicse vermeintliche oder laesüchliche Knse resgierenden Konzepuon des reflexiven Rechts als auch der populären Variante einer wautopoieuschen Selbsisteuerung ${ }^{*}$. Dicsen Zusammenliang übersıehı $m$. E. dic ohne aellere Argumente auskommende Keıcik an Nahamowirz von Erhard Blankenburg: Dic rechuliche Autonomie nordamerik ansscher Indianer-Reservate - ein Beispiel für die kolontsierende Wirkung reflexiven Reches. In: Zeisschrift für Rechissoziologie 1/1986, S. s3 ff. (53). Daß man die funkuanale Systemcheorie nicht obne weiveres als Apologie des freien Markecs lesen darf, subbe auf einem anderen Blats. Weiteres empirisches Material lindet sich bet Gerd Winter/Johann Unger: Interventionisusches und fexibles Verwaltungsrecht. D2s Besspiel Zweckentfremdungsverbot für Wohnraum. In: Krutusche Justiz 4/1984, S. 394 If.; Klaus Tonner: Verbrauchertecht und Selbseregulicrungskonzepec. In: Kritische Jusiz 2/1989, S. 107 If.

Ss Renatc Mayntz: Implementation polituscher Programme - empinsche Forschungsberichee. Konigstcin/ Ts. 1980 .

89 Niklas Luhmann: Poliusche Theoric, a. a. O., S. 96. 
den Fall eines für sie ungünscigen Ausgangs »Investicionseinschränkungen in Aussicht» stellt. Die Energiewirsschaft mache dem Geserzgeber und der Bevölkerung Angst, daß bei der Abschaffung der Atomenergie die Lichter und die Arbeitspläzze ausgingen. Sie machr die Angst - dies als Hinweis auf die politische Perspekrive, aus der das Problem systemtheoretisch gesehen wird-, es wird also die Angst gemacht, die dann denen, die sie haben solien und auch tatsächlich bekommen, von der Systemtheorie - genauer: von ihrem Schulenhaupr - auch noch mahnend vorgehalten wird, weil sie »zuviel Resonanz « auf das Umweltproblem erzeuge ${ }^{90}$;

"Angst widersteht jeder Kricik der reinen Vernunft Sie ist das modeme Apriori - nicht empirisch, sondern transzendental. Sie ist das Prinzip, das nicht versagt, wenn alle Prinzipien versagen. Sie ist ein ,Eigenbehavior, das alle rekursiven Teses überdauer. Man kann ihr eine große polirische und moralische Zukunft voraussagen. Ein Glück nur, daß die Rhetorik der Angst wahrscheinlich nicht in der Lage ist, wirkliche Angst zu erzeugen. Sie bleibt ein Störfaktor im sozialen System. $a^{9 t}$

Diesmal allerdings bleiben die Urheber niche in der Anonymität der Abstraktion:

"Das gesellschartliche Problem liegt allerdings weniger in der psychischen Realizät von Angst als in ihrer kommunikaziven Aktualität. Wenn Angst kommuniziert wird und im Koommunikationsprozeß nicht bestritten werden kann, gewinnt sic cine moralische Existenz. Sie macht es zur Pflicht, sich Sorgen zu machen, und zum Recht, Anteilnahme an Befurchtungen zu erwarten und Maßnahmen zur Abwendung der Gefahren zu fordern. Die ökologisch Besorgten rüsten daber niche nur, wie einst Noah, ihre eigenc Arche mit den für die spätere Evolution generisch notwendigen Materialien. Sie werden zu Warntätern - mit ali den moralischen Risiken, die das implizien. Die ökologische Kommunikaxion wird auf diese Weise über Angst mit Moral aufgeladen, und Kontroversen werden wcgen ihres polemogenen Ursprings unentscheidbar. Erst die Zukunft könnte zeigen, ob die Angst berechtigt gewesen war, aber die Zukunft konsutuiert sich in jeder Gegenwart neu.

Gegenüber einer Moral, die angstbezogene Unterscheidungen propagien, haben theoretische Analysen einen schweren Stand.... $\alpha^{91}$

Wir sollen also nicht soviel Angst haben ${ }^{93}$ bzw. nicht soviel darüber reden, besonders wenn wir gar keine haben. ${ }^{94}$ Dic Ausgangsannahme der Systemcheorie von der Ohnmache des Wohlfahrts(= Steuerungs)staates scheint damit ausreichend belegt. Dies allerdings nicht so, wie die Theorie es sich vorscellt, sondern in einem genau entgegengesetzcen Sinne: Die Probleme rühren nicht durchweg aus dem Fehlen eines Steuerungszentrums, der fehlenden Möglichkeit also, gegebene Befehlc wirksam durchzusetzen, weil sie sich an den Grenzen der Subsysteme brechen bzw. in aronym-diffusen Incerdependenz-Beziehungen foigenlos oder in einem nicht gewollten Sinn: folgenreich, verhallen. Gut organisierte und entsprechend leicht identifizierbare Interessen blockieren (in unseren Fällen dürfte man getrost sagen: erpressen) ein insoweit vorhandenes politisches Zentrum. Aber solche Überlegungen, nach denen eine gesellschaftliche Macht erfolgreich auf ein System (Politik) einwirkt, gelten dem Systemfunkrionalismus als das Erbe eines antiquierten Kausaldenkens, das sich mir dem ig. Jahrhundert überlebe hat. Im systemfunktionalen Denken - ein bäufig wiederholter Vorwurf - kommen "Interessen", „Herrschafts $\alpha$ beziehungen und ähnliche moralische und somit verbrauchte Begriffe nicht vor.

Eine Posizion, die jede Eigenlogik der Subsysteme als selbständigen Problemfaktor leugnet, käme allerdings in Schwierigkeiten. Sie könnte z. B. das Schicksal der

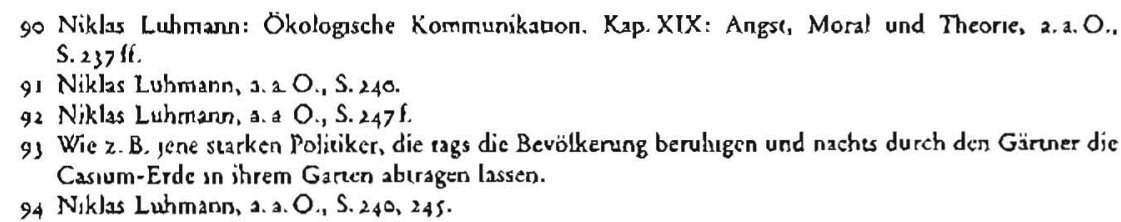


Juristenausbildungsreform nicht erklären. Für ihr zumindest teilweises Fehlschlagen lassen sich zwar eine Fülle sehr konkreter Interessen benennen, die sich durch einen Reformerfolg hätten bedroht fühlen können. Schon die Tatsache aber, daß die Reformmodelle unter unterschiedlichen politischen Bedingungen mit ähnlichen Schwierigkeicen zu kämpfen hatren, läßt es wenig sinnvoll erscheinen, die Erklärung \{ür das Scheicern der Reform auf die Aufzählung ihrer Gegner zu beschränken. Die Reformstrategie hat die spezifische Eigenexistenz der beteiligten Systeme: Wissenschaft, "Praxis" (= fustiz, Verwaltung, Wirtschafe) und Politik, nicht ausreichend in Rechnung gestelit - nicht zuletzr auch das Problem, um das es auch in der Autopoiesis des Rechts geht: der Autonomie des Rechts, die zwar in einem logischmethodischen Sinne abgewiesen werden kann, aber gleichwohl als soziologisches Phänomen real exisciert." ${ }^{\text {'s }}$

\subsection{Die Autopoiesis als Selbsterzengung des Recbes}

Wie läßr sich das Recht unter den Bedingungen einer entweder fehlenden oder unwirksamen "Sreuerungszentrale «eschreiben? Die Anrwor such die systemtheoretische Rechtssoziologie in einer weiteren Forschreibung ihres Konzepts von der Ausdifferenzierung der Funkrionssysteme, also in unserem Zusammenhang in einer weiceren Ausdifferenzierung des Rechts. Die Gesellschaft muß sich niche nur damit abfinden, sondern ist im Gegenteil darauf angewiesen, daß ihre Probleme nur noch "arbeitsteilig " in autonomen Teilsystemen erledigt werden können, die nach eigenen, nicht "von außen « direkt beeinflußbaren Kriterien arbeiten. Sie alle haben

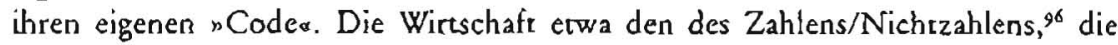
Religion den der Immanenz/Transzendenz, die Wissenschaft den der Wahtheic/ Unwahrheit, und das Recht orientiert sich ausschließlich am Code Reche/Unrecht. Diese jeweils systemeigene Sprache läßt sich nicbt in die Sprache des anderen Systems übersetzen. Es kann insoweit keine Direkt-Kommunikation zwischen den Systemen geben. Diese Autonomie der Funktionssysteme birgt Probleme in sich, die letzclich die Existenz der Gesellschaft selbst in Frage stellen können. In diesem Sinne denkr auch die Systemrheorie durchaus »bestandskritisch - wie immer man zu den vielen politischen Botschaften stehen mag, die sie in ibren Abstraktionen verhüllt. Jeder Eingriff, der diese Eigengesetzlichkeit des jeweiligen Systems nicht beachret, läuft Gefahr, wirkungslos zu bieiben oder unkontrollierbare Effekte zu erzeugen. Ein in dieser Weise munsensibler « politischer Befehl an das Rechussystem wist entweder irrelevant oder hat desintegrierende Wirkungen für den gesellschaftichen Lebensbereich oder aber desintegrierende Wirkungen auf das regulatorische Reche selber «"

Wie stellt sich nun die Autopoiesis des Rechts dar, das aiso nicht mehr als verläßliches Ausführungsorgan irgendwelcher Mächte begriffen werden darf? Wie eine Amöbe reproduziert es sich selbst. Im Unterschied zu ihr, die Geburt, Leben und Tod kennt, muß sich das Rechrssystem kontinuierlich, sozusagen von Sekunde zu Sekunde, perpervieren. Es bedient sich dabei ausschließlich seiner eigenen Elemente, hält sich also nicht durch permanente Auscauschprozesse mit der Umwelt in Gang. Dabei soll es sich nicht lediglich um einen Prozeß der Selbstorganisation

95 Das Thema habe ach in bezug auf dieses Problem bebandelt in Joachum Nacke: Die Juriseenausbildungsreform als Geserzgebungsexperiment. Der Betrrag erschexnt demnächst un den Schrifien der Veremigung für Rechessoziologic.

$96 \mathrm{Vgl}$. zum folgenden unter den Abschniteen des jeweils behandelten Funkuonssystems: NikJas Lubmann: Ökologrsche Kommunikatıon, a.a. O.

97 Gunther Teubner: Verrechtlichung, 2.1.O., S. j16. 
handeln, wie man ihn schon an lernenden offenen Systemen beschreiben konnte. Vielmehr geht man jeczt von einer umfassenden Selbscorganisation des Systems aus, die nicht nur seine Strukturen betrifft. "Vielmehr ist von selbstreferentiellen Systemen die Rede, die jede Art von Einheir, die sie benötigen und́ verwenden, selbst herstellen: Auch die Einheit des Systems selbst und auch die Einheit derjenigen Elemente (z. B. Handlungen), aus denen das System besteht. $\alpha^{98}$ Das autopoietische Rechr nimme in der weireren Diskussion sogar handgreifliche Gestaht an: Es entwickelt ein Gedächtnis, ${ }^{99}$ prägt ein Nervensystem aus, das, statt den einfachen linearen Ursache-Wirkungs-Ketren zu folgen, in geschlossenen Netzwerken ${ }^{100}$ sich selbst beobachter, in basaler Zirkularität - ein Zentralbegriff der Auropoiese - obne vorschreibbare Zwecke und Ziele sich beständig selbst fortzeugend am Leben hält.

\subsection{Der Dualismus des autopoietischen Rechts: Normative Geschlossenbeit- kogntive Offenheit}

Spätestens bei der Lektüre des letzten Satzes wird Herr Alfred Krause vom Deutschen Beamtenbund Unmut verspüren. Wird hier einmal mehr der rechtsstaatlichen Verwaltung Hohn gesprochen, die sich angeblich nur am Leben hält, indem sie von selbstproduzierten Problemen zehrt? Welches Zerrbild - wird der Deutsche Richterbund nachseczen - zeichnen gewisse Soziologen wieder einmal von der Justiz, die kein Ohr für die Sorgen des Bürgers haben soll, wohl aber in selbstbeobachrender Nabelschau um selbsterzeugte Probleme kreisen soll!

Aber der Verdacht ist unbegründer. Hier ist keine Diffamierungssoziologie am Werke. Die gibr es nämlich nicht mehr. Hubert Rotrleuthner iss in die Jahre gekommen, der väterliche Herr Blankenburg war schon immer dort, und die Frankfurter sind konvertiert oder schmollen in der Eremitage. Auch Niklas Luhmann hat sich mit dem System noch nicht überworfen. In der zeitgenössischen Rechtssoziologie geht es rundum staatstragend zu. Das modische Theoriedesign schreibr Nadelfiiz vor. Hier wird keine vorschnelle »Ideologiekritik $\propto$ mehr geübe schon der Begriff nimmt sich in der verchromten Sprachwelt der Systemtheorie aus wie eine ehrwürdige Anriquitär, die von den hier Tätigen freilich eher wie eine peinliche Hinterlassenschaft gemieden wird. Hier will keiner mehr irgend jemandern ans Leder. Mit Ausnahme der Grünen, die mit ihren fehlenden Schlipsen ${ }^{(0)}$ die Kleiderordnung entdifferenzieren und damit das Gesamtsystem, dessen Teil sie letzelich selbst sind und dem sie nichr entrinnen können, ${ }^{102}$ übermäßig in Schwingungen ${ }^{\text {os }}$ verseczen.

Natürlich ist auch im autopoietischen System die Justiz für das Volk und die Verwaltung für den Bürger da. Jene Leser haben nur zu früh die Geduld verloren. Das geschlossene, selbstreferentielle System ist nicht vom Autismus befallen. Es hat nicht nur ein Gedächrnis, mit dem es immer nur an sich denkt. Es hat auch - sehr versteckt freilich - Sinne, die es mit der Außenrvelt verbinden, und es gibt Medien, über die es mit seiner Umwelt kommunizieren kann. Es ist, so die neue Vorstellung, geschlossen und offen zugleich.

Um diesen scheinbaren Widerspruch zu verstehen, muß man noch einmal einen

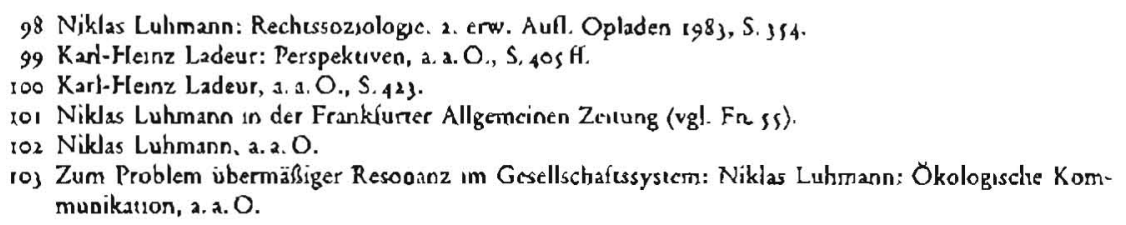


rieferen Blick in das Luhmannsche Theoriegebäude werfen. In ihm hat der systemtheoretische Troß - oder sagen wir: die Vorhur - mittlenweile Quartier genommen, so daß es für die deursehe Szene vorbildlich wirken wird. Luhmann also sagt: Alles Recht ist ein normativ geschlossenes, aber kognitiv offenes System. ${ }^{104}$

Dazu der Versuch einer kurzen Erläuterung. Jedes soziale System - demnach auch das Recht - besteht aus Kommunikationen. Sic bilden die Elemente ${ }^{10 s}$ des Systems nicht erwa Menschen, Gerichtsgebäude, Barecte. Diese sind also keine Bestandteile, sondern gehören zur Umwelt des Systems. In der Theorie kommen überhaupt keine leibhaftigen Menschen, sondern nur psychische Systeme, Bewußtseinssysteme u. dgl. vor. Dieser Umstand wird der Theorie stets zu Unrecht verübelt. Auch in anderen "Sinnsystemen - etrva in der Rechtscheorie des Sozialisten Hans Kelsen kommr kein Mensch als solcher vor. Diese Feinheiten müssen hier zurückstehen. Wesentlich ist nur noch eine zweite Einsicht: Das Rechtssystem ist nicht auf das kommunikarive Geschehen beschränkt, wie es in Justiz, Anwalsschaft, Gesetzgebung ${ }^{106}$ und Behörden organisiert verläuft. Es umfaßt kommunikative Handlungen, die sich in der Gesellschaft am Code Recht/Unrecht orientieren.

\subsection{Die Geschichte vom Erbonkel - autopoietisch erzäblt}

Das umfassend gedachte Rechtssystem wird also normativ geschlossen und zugleich kognitiv offen gesehen. Diesen Gedanken veranschauliche man sich am besten an einem Fall, z. B. dem des Erbonkels, der beim Spazierengehen tor neben mir umfällt. Ich nehme ihm die goldene Uhr ab, denn sie ist nun mein. Juristisch becrachtec hat sich in diesem Augenblick viel ereignet. Das Grundbuch der Gemeinde ist mit dem letzten Atemzug, den der Onkel auch als Immobilieneigentümer tac, unrichtig geworden. Ich bin in seine Rechtsstellung urnfassend eingerückt ( $\$ 1922 \mathrm{BGB}$ ), gehe also als vermögender Mann weiter, muß aber auch für die Schulden aufkommen usw. Für den Systemcheoreciker ist bis zu diesem Zeitpunkt das Geschehen noch nicht eindeutig idencifizierbar. Es hat noch keine Kommunikation stattgefunden, die eine Zuordnung erlaubte, welchem Teil- oder Subsystem das Ereignis zugerechnet werden könnte. Das Gespräch mit dem Onkel - ich hatte gerade durchblicken lassen, daß ich sein Erbe später einmal in die neue Stiftung der Grünen einbringen werde - ist abgebrochen. Das Rechessystem lebr sozusagen erst in dem Augenblick auf, in dem sich eine rechtlich folgenreiche, also diesbezüglich erwarcungsbildende Kommunikation ergibe, die sich am Code Reche/Unrecht orientiert. Das wäre z. B. der Fall, wenn cine Passantin mir die Uhr screicig machen wollte, ich im Hinweis auf meinen Heredizär-Status mich zur Wehr setzte, sie mich festhielte, die Polizei riefe usw. Auch wenn dies wortlos geschähe: Es werden - auch nonverbal - Informationen migeteilt und entsprechende Erwartungen gebilder, gleichviel, ob sie denen des anderen entsprechen oder nicht. Notfalls sieht man sich vor Gericht wieder. Die Kommunikation ist in jedem Fall am Code RechuUnrecht orientiert, und die Bereiligten stellen ihre Erwartungen dementsprechend ein. Über dem Onkel perpetuiert sich das System des gesellschaftichen Rechts.

104 Niklas Luhmann: Rechrssoziologie. 2. erw. Aufl. Opladen 1983, S. 354 ff.

105 Diese Aussage isı nach dem letzren Stand der Theone schon zu grobköraig. Vgl. Niklas Luhmann: Die Einheıt des Rechussystems, a. a. O.

roó Die Geserzgebung n'urd also nicht etwa der Politik, sondem dem Rechtssystem zugerechnet. Diese ketneswegs selbstverständliche Entschcidung, dic Politik auf Parceipolicik und, grob gesagt: Posecnschiebere beschränkt (vgl. Niklas Luhmann: Ókologaschic Kommunikauon, a. a. O., S. 167 ff.), ust (üj die rechuliche Autoporesis der Systemcheone ubertebenswichug, weil nur so die Autonome des Rechis gegenüber der Policik behauper werden kann und nicht erwa - wie busher üblich - als enn reiucs Reches anwendungsproblem (jurisusch: "Unabhängxgkeit der Rechispllege*) behandele warden muß. 
Wie wird nun im autopoiecischen Rechtssystem darüber entschieden, was Unrecht ist und was Recht? Die herkömmliche Sicht harte damit keine prinzipiellen Schwjerigkeiten. Über diese Frage entscheidet derjenige, der die Macht dazu hat. Über die Frage von Recht und Unrecht entscheidet also das Parlament, ein Diktator, die Veruragsparteien in den Grenzen iturer Autonomie, die Rechrsanwender, soweit sie ihre Interpretationsberrschaft bei der Exegese von Rechtstexten nutzen usw. Es verbleibt dann noch die Frage, die der Soziologie ihr kricisches Potential verleihr bzw. verlieh: welche Kräfte jenen Rechtssetzern catsächlich die Feder führen.

Diese grobschlächtige Gegenständlichkeit ist für die Systemtheorie nicht verdaulich. Auf dieser für sie simplifizierenden Ebene, auf der man nur auf falschen Fährten nach Hinternännern, letzten Ursachen und Verantwortlichkeiten, die es allesamt nicht gibt, suchen kann, läßt sich die Selbsterzeugung von Recht nicht darstellen. Eine in diesem Kausaldenken befangene Rechtssoziologie - so der sters erneuerte Einwand - verführt zu vorschneller Kricik schon deshalb, weil dieses instrumentelle Rechesverständnis die Autonomie des Rechts niche erfassen kann. Das autopoietische Recht ist vielmehr ein normativ geschlossenes System, weil über die Normativität, das Gesolltsein, auf das sich die Erwartungen hin bilden, ausschließlich im Rechtssystem selbst entschieden werden kann. Das System kann nicht aus Ereignissen in der Umwelt darauf schließen, was sein soll. Hier findet sich also der von Kelsen vielfach variierte Gedanke von der wechselseitigen Unableitbarkeit von Sein und Sollen wieder. ${ }^{107}$ Normativität kann also nicht aus der Umwelt bezogen werden, sondern wird im System selbst erzeugt. Es reproduziert sich also forlaufend in der permanenten Produktion von Normativität.

"Das rechuliche Gesolltsein symbolisier die Einheil und Geschlossenheir des Systems. Es gibe kein Recht außerhalb des Rechts, also im Verhältnis zur gesellschaftlichen Umweit des Systems weder Input noch Output von Recht. Mit anderen Wornen: die gesellschaftliche Funkuion des Rechus wird ausschließlich im Rechissystem wahrgenommen und sonst nirgendwo. ${ }^{108}$

Im eirzelnen führt dieser Gedanke in Taurologien und Paradoxien. Z. B. in den Fällen, in denen es darum geht, sob die Unterscheidung von Rechr und Unrechr mic dem Recht oder mit Unrecht eingeführt wird. ${ }^{109}$ Anders als Kelsen, der entsprechende Probleme in einer linearen, logisch-deduktiven Normenhierarchie aufzulösen suchte, will die Autopoiesis-Konzepcion dieser Sackgasse ausweichen, die irgendwo in der Annahme einer hypothetischen Grundnorm und für den Juristen z. B. in Art. 79 III GG, der sog. Ewigkeitsklausel, enden muß. Die Systemtheorie sicht jene Paradoxieo und Taucologien so ähnlich wie der niederländische Zeichner Maurivius C. Escher ${ }^{10}$ : Auf seinen Bildern geht man ständig eine Treppe hinunter, um sters wieder oben anzukommen. Hier wird also das "Endlose Geflochtene Band ergriffen, von dem schon die Rede war. Der Vorgang der Zeugung von Normativität aus Normen kennt jene Trennung verschiedener Ebenen nicht mehr, wie man sie sich im Bilde einer Normpyramide oder einer Hierarchie von Rechtsquellen zu veranschaulichen suchce. An ihre Stelle treten sverwickelte Hierarchien«, "selesame Schleifen « und andere kybernetische Meraphern, denen allesamt gemein ist, daß sie keine Línearität und keine Kausalizät, keinen Anfang und kein Ende

107. Und auch sonst wirk! die Konzepuon streckenwerse pre eine Übersetzung der Kelsenschen Rechistheorie in die systemcheoreusche Rochusoziologie. Zu dieser Parallele: Harst Dreier: Hans Kelsen und NikJas Luhmann: Posıuvirät des Reches aus rechesw'ssenschaftlicher und systemcheoreascher Perspek. uve. In: Rechestheorie 1983, S. 4 ig ff.

108 Niklss Luhmann: Dic sozıologische Betrachung des Rechts, a.a. O., S. 20.

109 A. 2. O., S. 16.

1 so Seine paradoxen Bilder finden sıch in: Douglas R. Hofstader: Ein Endloses Gtflochtenes Band, a. a. O. 
kennen. " Die Expedicion zu den Urgründen des Rechts mag sich noch so sorgfältig rüsten - sie wird nie ankommen.

Dieses normativ geschlossene System ist zugleich nkognitiv offen «. Als System, das ein "Innen* kennc, muß es begriffsnotwendig auch ein "Außen $\alpha$, also eine Umwelt haben. Das System braucht diese Umwelt sogar. Denn es muß zur Kommunikation auch Anlässe geben. Ereignisse in der Umwelt sind gewissermaßen Rohmaterial einer möglichen Kommunikation. Welches Ereignis sich die Kommunikation als Anlaß sucht und in welchem Code sie verläuft, darüber entscheidet sie als System selber. In jedem Fall muß sie für jene Ereignisse kogniriv offen sein, weil Kommunikation sonsr gar nicht zustande käme. Eine Verwaltung kann sich - das war ja das Mißverständnis des Deutschen Beamtenbundes - nicht nur von eigenen Problemen nähren.

Für welche Ereignisse in der Umwelt das Rechtssystem seine Augen offen halten und auf welche Anlässe es reagieren muß, ist in den systemeigenen Programmen gespeichert: Geserze, Verträge, Rechtsprechungsroutinen, herrschende Meinungen, Rechtsdogmatik. Für unseren Fall gilt $\$ 1922$ BGB: Herzinfarkt - Erbfall - Grundbuchberichtigung. Über die Entscheidung von Recht oder Unrecht kann in funktional differenzierten Gesellschaftssystemen nicht - wie im Faustrecht - spontan entschieden werden - jedenfalls nicht durchgängig und auf Dauer. Dzs würde dem System jede Möglichkeit nehmen zu lemen, d.h. Informationen aus der Umwelt strukcurell zu verarbeiten. "Durch die Differenzierung von Codierung und Programmierung gewinnt ein System also die Möglichkeir, als geschlossenes und offenes System zugleich zu operieren. u $^{112}$

\subsection{Die Autonomie der Funktionssysteme als Problem der Gesellschaft}

Das Grundmustex, das hier am Rechsssystem demonstriert wurde, läßt sich auf jedes andere Funktionssystem übertragen. Diese Einsicht erklärt vielleicht die nicht abreißende Produktion der Systemtheoreriker und hilft unserem dadurch etwas angeschlagenen Selbstbewußrsein auf. Abgesehen davon kann man jetzr etwas klarer sehen, weshalb die Systemtheorie nicht an die zentrale Planung im Wohlfahrtsstaar glauben kann. Das Recht kann sich z. B. in bezug auf Umwelcprobleme nur nach Maßgabe seiner Möglichkeiten verhalten. Es kann darauf reagieren, indem es für schwefelnde Schlore erwa eine Entgifnungsanlage vorschreibt und für die Nichtbeachrung ein Bußgeld vorsieht. Es kommuniziert hier also im Code Recht/Unrecht. Mit diesem Code kann es aber auf das System Wirtschaft nicht unmittelbar einwirken. Dieses Syscem kommuniziert nach dem Code Zahien/Nichtzahlen, denkt also in Preisen. Es kann sich zu Umweltproblemen ausschließlich auf dieser Ebene verhalten. Für die Wirtschafr ist die neue bußgeldbewehre Vorschrift zwar ein externes "Datum" - ein Geschehen in ihrer Umweic, das registrier, aber ausschließlich nach systemeigenen Kriterien verarbeilet wird. Sie kalkuliert nämlich, ob die Zahlung eines Bußgeldes nicht vorteilhafter sein könnte als der Einbau einer teuren Encschwefelungsanlage. Trufft sie die Encscheidung nach diesen Kriterien, handelt sie als wirzscbaftliches System. Kommt es hingegen zum gerichtlichen Streit bis hin zum Einbau einer Anlage im Wege der Ersaczvornahme durch das zuständige Umweltminiscerium, orientieren sich die Beteiligten nicht nach dem Code Zahlen/Nichtzahlen, sondern an dem von Recht/Unrechr, womit sich in diesem. Falle das Rechtssyscem reproduziert. Dieser Weg isc zwar denkbar, wird aber, wenn

1 I NikJas Luhmann: Die sozıologische Betracheung des Rechts, 2.1. O.. S. 16. 112 NikJas Luhmann: Ökologische Kommunikatıon, a. a. O., S.gi. 
derartige Lösungen zur ständigen Übung zu werden drohen, die Möglichkeiten des Rechts auf Dauer überfordern. Es stellen sich dann vielleicht auch Probleme im Verhältnis zur Politik ein, z.B. wenn in der politischen Auseinandersetzung über das Thema nach dem Code: Parlamentsposten/keine Parlamenesposten kommuniziert wird.

\section{Die Rechessoziologie in seltsamen Schleifen}

Bestimmte Pendelbewegungen des vorstehenden Textes ergeben sich aus der Ambivalenz der cheorecischen Abstraktion selbst, die in der systemtheoretischen Rechtssoziologie auf die Spitze getrieben ist. Absiraktion kann eimerseits jede Gegenständlichkeit verschwinden lassen und damit einer auf Anschaulichkeis bedachten Kritik ihr Objekt nehmen. Auf dieser Ebene wird die systemtheorecische Variante der Rechessoziologie heute von ihren Krivikern vor allem wahrgenommen: eine im monomanen Abstraktionsrausch dahinrasende Rhetorik, die gleichwohl mir missionarischem Eifer gcgen irgendetwas anzuschreiben scheint. Was das letztere betrifft, so erkennt man in der Tar in der politischen Physiognomie dieser Theorie, vor allem in der Fassung ihres Schulenhauptes, vertrauce Züge. In diesern Text war es, stellvertresend für anderes, das Verbälenis der Theorie zu alcernativen polizischen Bewegungen. Josef Kardinal Hölfner hält die Grünen für unwähtbar, die konservative Jurisprudenz erkennt sie als verfassungswidrig, und Niklas Luhmann betrachtet sie als einen Störfaktor im politischen System. Der eine beruft sich auf die Bibel, die anderen auf das Grundgesetz und der dritte auf den Zweiren Hauptsatz der Thermodynarnik. Haben Theologie, Rechsswissenschaft und Soziologie unter dem Dach einer langersehnten Universalwissenschaft zusammengefunden, daß sie nun mir einer Zunge sprechen können? Man mag uns kleingläubig schelten, wenn wir in diesem Punkt noch etwas zögern.

Aber diese Form der Ideologieproduktion ist nur eine mögliche, nicht aber eine zwingende Konsequenz der Abstraktion. Abstrakrion muß Kritik nicht leerlaufen lassen, sie kann im Gegenteil zur Kricik erst befähigen. Jede gure Karikatur beweist das. Sie ist dann gelungen, wenn sie sich von der Gegenständlichkeir ihres Objekts löst. Durch konsequente Abstraktion, Verzicht auf Maßstabtreue und eigenwillige Interpretation verallgemeinert sie krivisch das, wofür ihr konkreter Gegenstand nur den Anlaß liefert und was die pingelige Fleißarbeir eines Realisten gerade verdecken würde. Der allwissende Miniscer schnurre so auf den doppelzüngigen Quarschkopf zusammen, der für viele seiner Gattung steht.

Und eben dieses kritische Potential von Abstraktion ist in der Theorie vor allem Luhmanns angelegt. Man kann die funktionaliscische Rechrssoziologie als vollständige Descrukrion und Bestärigung der Juristenideologie zugleich lesen. Sie beschreibr als Wissenschaft, aus der Oprik der Fremdbeobachcung also, wie die praktische Rechtsdogmatik als autopoietisches System in seltsam gedrehten Schleifen in sich kreist, ohne daß die Beteiligten es merken. Aus ständig wechselnder Perspektive sehen wir - historisch reich illustriert - das Recht in den eigentümlichsten Paradoxien sich winden. Aber diese Fremdbeobachtung versagr sich und anderen jeden Anspruch auf Besserwisserei." Als Wissenschafi bewege sie sich in eigcnen gescilossenen Bahnen und damit in eigenen Paradoxien, die es ihr verweh- 
ren, dem praktischen Recht, das ebenfalls nur sich selbst beobachten kann, ihren Kurs aufzuzwingen. Was wäre auch damit gewonnen, wenn man den Achterbahnfahrern mit ihren flatternden Roben beständig nachriefe, daß sie und ihre Argumentation mal wieder auf dem Kopfe stehen?

Vieles spricht dafür, daß die Rechtssoziologie mit der Autopoiesis selbst gerade diesen Zustand durchfährt. In den Zwängen ihrer eigenen. Theorie gefangen und der Suggestion ihrer plakativen Metaphern erlegen, saust sie dahin, und wir hören, wie urs Karl-Heinz Ladeur im Vorbeifahren zuruft, nicht vorschnell zu urteilen, sondem sorgfälrig abzuwägen. Aber:

„Ein dynamisches Theorie-Modell der Abwägung muß sich aber auf die Temporalisierung auch der sırukturellen Rationaliät der dadurch institutionalisierten Form der Optimierung von Informationsverarbeitung erstrecken, indem es den einheitlichen Sprach- und Arikulacionsraum des Modells in einen topologisch variablen Projekcionsraum diskontinuierlicher, zeirgleicher und zeitverschiedener Arrangements von Möglichkeiten überführt. ${ }^{1 " .}$

Wer bei dieser Abwägung ein wenig ins Schwanken geraten ist, wird es gespürt haben: Die Rechtskritik wird es künfrig schwerer haben. Sie muß nicht nur - mühsam genug - die Dogmatik entschlüsseln, sondern auch noch die Rechrssoziologie/theorie, die sich mit ihr beschäftigt. Diese Wissenschaft ist zunehmend mit der Lösung von Rätseln ausgefüllt, die sie sich selbst aufgibt. Die Autopoiesis ist insoweic das Resultat einer präzisen Selbstbeschreibung des Systems.

114 Karl-Heinz Ladcur: *Abwägung*, 2. 2 O, 5.2 219.

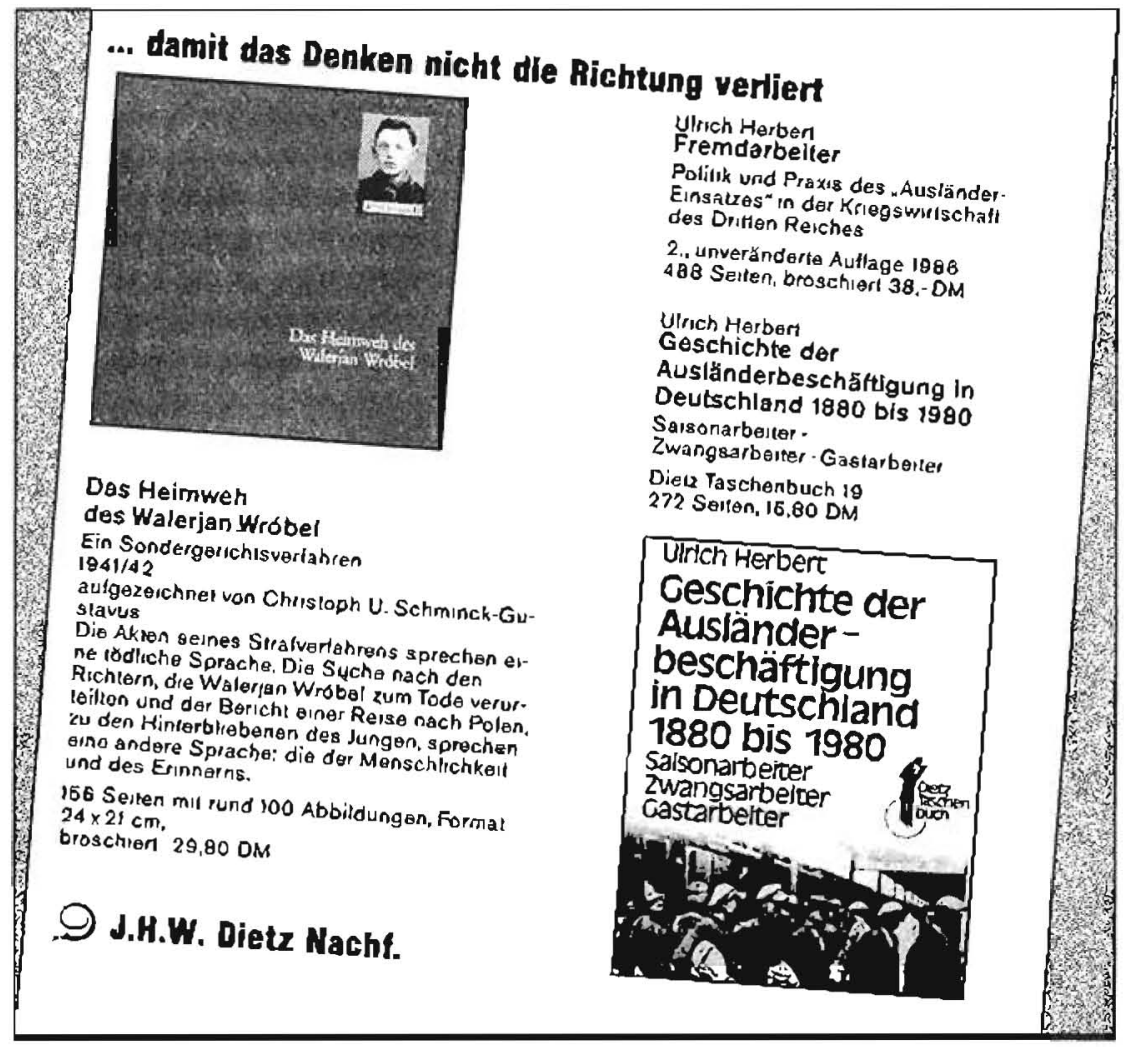

